\title{
Recent Advances in the Theory and Application of Lie Pseudo-Groups
}

\author{
Peter J. Olver ${ }^{\dagger}$ \\ School of Mathematics \\ University of Minnesota \\ Minneapolis, MN 55455 \\ olver@math.umn.edu \\ http://www. math. umn.edu/ olver
}

\begin{abstract}
This paper surveys several new developments in the analysis of Lie pseudogroups and their actions on submanifolds. The main themes are direct construction of Maurer-Cartan forms and structure equations, and the use of equivariant moving frames to analyze the algebra of differential invariants and invariant differential forms, including generators, commutation relations, and syzygies.
\end{abstract}

\section{Introduction.}

Inspired by Galois' introduction of group theory to solve polynomial equations, Lie founded his remarkable theory of transformation groups for the purpose of analyzing and solving differential equations. In Lie's time, abstract groups were as yet unknown, and hence he made no significant distinction between finite-dimensional and infinitedimensional group actions. However, since then the two theories have evolved in radically different directions. Through the work of Cartan, Weyl, Schreier and Chevalley, the modern abstract theory of finite-dimensional Lie groups has become well-established and widely used throughout mathematics and its physical applications. In contrast, the theory of infinite-dimensional pseudo-groups remains in a surprisingly primitive state, and there is still no generally accepted object to represent an abstract Lie pseudo-group.

$\dagger$ Supported in part by NSF Grant DMS 08-07317.

February 25, 2010 
The importance of Lie pseudo-groups is accentuated by their appearance in a broad range of physical and geometrical contexts, including gauge theories in physics, [7], Hamiltonian mechanics, symplectic and Poisson geometry, $[\mathbf{5 4}, \mathbf{6 4}]$, conformal geometry and conformal field theory, $[\mathbf{2 4 , 2 6}]$, the geometry of real hypersurfaces $[\mathbf{2 1}]$, foliations and characteristic classes, $[\mathbf{2 9}, \mathbf{7 9}]$, symmetry groups of both linear and nonlinear partial differential equations, [64], Vessiot's method of group splitting for producing explicit solutions to nonlinear partial differential equations, $[\mathbf{5 3}, \mathbf{6 2}, \mathbf{7 7}, \mathbf{9 3}]$, fluid and plasma mechanics, $[\mathbf{3}, \mathbf{1 3}, \mathbf{6 4}]$, meteorology, $[\mathbf{6}, \mathbf{8 2}]$, integrable (soliton) equations, [23], equivalence problem for differential operators and Laplace invariants of hyperbolic systems, [85], mathematical morphology and computer vision, [84,95], and geometric numerical integration, [55]. And keep in mind that any sufficiently regular local Lie group action qualifies as a Lie pseudo-group.

For historical contributions to the subject, we refer the reader to the original papers of Lie, Medolaghi, Tresse, and Vessiot, $[45,46,58,89,93]$, for the classical theory of pseudogroups, to Cartan, $[\mathbf{1 6}, \mathbf{1 8}]$, for their reformulation in terms of exterior differential systems, and $[25,36,37,43,44,47,61,78,81,86,87,88]$ for a variety of modern approaches. Recent advances began with [27], that proposed a new approach to the classical theory of moving frames for general transformation groups. In the case of finite-dimensional Lie group actions, the reformulation of a moving frame, $[\mathbf{1 4}, \mathbf{3 0}]$, as an equivariant map back to the Lie group, [28], proved to be amazingly powerful, sparking a host of new tools, new results, and new applications, including complete classifications of differential invariants and their syzygies, $[\mathbf{3 1}, \mathbf{6 9}, \mathbf{7 1}$ ], equivalence, symmetry, and rigidity properties of submanifolds, [28], computation of symmetry groups and classification of partial differential equations, $[49,59]$, invariant signatures in computer vision, $[4,8, \mathbf{1 2}, 67]$, joint invariants and joint differential invariants $[\mathbf{9}, \mathbf{6 7}]$, rational and algebraic invariants of algebraic group actions $[\mathbf{3 2}, \mathbf{3 3}]$, invariant numerical algorithms $[\mathbf{3 8}, \mathbf{6 8}, \mathbf{9 5}]$, classical invariant theory $[\mathbf{5}, \mathbf{6 6}]$, Poisson geometry and solitons $[\mathbf{5 0}, \mathbf{5 1}, \mathbf{5 2}]$, the calculus of variations and geometric flows, $[\mathbf{3 9}, \mathbf{7 0}]$, invariants and covariants of Killing tensors, with applications to general relativity, separation of variables, and Hamiltonian systems, [56, 57], and invariants of Lie algebras with applications in quantum mechanics, [10]. Subsequently, building on the examples presented in [27], a comparable moving frame theory for general Lie pseudo-group actions was established, $[\mathbf{7 2}, \mathbf{7 3}, \mathbf{7 4}]$, and applied to several significant examples, $[\mathbf{1 9}, \mathbf{2 0}]$.

The present survey begins with the basic definitions of pseudo-group and Lie pseudogroup, showing that any regular pseudo-group has a canonical Lie completion that possesses exactly the same local geometry and invariants, $[\mathbf{3 4}, \mathbf{3 5}]$. The Maurer-Cartan forms of a Lie pseudo-group are explicitly constructed as invariant differential forms on the infinite pseudo-group jet bundle, [72]. Moreover, the structure equations are found by restricting the explicit diffeomorphism structure equations to the kernel of a linear algebraic system directly related to the linearized determining equations for the pseudo-group's infinitesimal generators. A large number of examples arise as symmetry groups of differential equations, and we provide a quick review of the classical Lie infinitesimal method of calculating symmetry groups, [64], and then show how, using the preceding result, the structure of the symmetry group of a system of differential equations can be directly found without integration of the determining equations, $[\mathbf{1 9}, \mathbf{7 2}]$. We then review the extension of the 
equivariant moving frame theory to pseudo-group actions on jets of submanifolds, leading to an algorithmic procedure for constructing the differential invariants and invariant differential forms, $[\mathbf{2 0}, \mathbf{7 3}]$. The final section reviews how the moving frame calculus is used to determine the structure of the differential invariant algebra, including classification of generators, commutation relations, and differential syzygies, [74]. The mathematical machinery underlying all these results is the variational bicomplex on infinite order jet spaces, $[\mathbf{1}, \mathbf{2}, \mathbf{3 9}, \mathbf{9 0}]$, which also lies at the heart of the modern geometric approach to differential equations, variational problems, symmetries and conservation laws, characteristic classes, and elsewhere.

\section{Pseudo-Groups and Lie Pseudo-Groups.}

Let us begin by recalling the basic definitions. To avoid technical complications, we will work in the analytic category throughout. Thus, by a local diffeomorphism of an analytic manifold $M$ we mean a one-to-one analytic map $\phi: U \rightarrow V$ defined on open subsets $U, V=\phi(U) \subseteq M$, with analytic inverse $\phi^{-1}: V \rightarrow U$. Adapting the constructions to smooth $\left(\mathrm{C}^{\infty}\right)$ pseudo-groups requires some additional care, as noted below.

Definition 2.1. A collection $\mathcal{G}$ of local diffeomorphisms of a manifold $M$ is called a pseudo-group if

- if $U \subset M$ is an open set and $\phi: U \rightarrow M$ is in $\mathcal{G}$, then $\phi \mid V \in \mathcal{G}$ for all open $V \subset U$.

- if $U_{\nu} \subset M$ are open subsets, $U=\cup_{\nu} U_{\nu}$, and $\phi: U \rightarrow M$ is a local diffeomorphism with $\phi \mid U_{\nu} \in \mathcal{G}$ for all $\nu$, then $\phi \in \mathcal{G}$.

- if $\phi: U \rightarrow M$ and $\psi: V \rightarrow M$ are two local diffeomorphisms belonging to $\mathcal{G}$ with $\phi(U) \subset V$, then $\psi \circ \phi \in \mathcal{G}$.

- if $\phi: U \rightarrow M$ is in $\mathcal{G}$, and $V=\phi(U)$, then $\phi^{-1}: V \rightarrow M$ is also in $\mathcal{G}$.

Note that the second and fourth requirements imply that $\mathcal{G}$ necessarily contains the identity diffeomorphism: $\mathbb{1}(z)=z$ for all $z \in M$. The simplest example is the collection of local analytic diffeomorphisms of an analytic manifold $M$, denoted $\mathcal{D}=\mathcal{D}(M)$. All others are sub-pseudo-groups thereof.

Ehresmann's geometric formalization of the calculus of Taylor polynomials and series through jet bundles, [25], was expressly introduced for analyzing pseudo-group actions. For each $0 \leq n \leq \infty$, let $\mathcal{D}^{(n)} \subset \mathrm{J}^{n}(M, M)$ denote the bundle formed by the $n^{\text {th }}$ order jets of local diffeomorphisms. In particular, $\mathcal{D}^{(0)}=M \times M$, while for $n \geq 1$, by the Inverse Function Theorem, $\mathcal{D}^{(n)}$ is characterized by the non-vanishing of the Jacobian determinant. The $n$ jet of a local diffeomorphism $\phi$ forms a $m$-dimensional submanifold $\mathrm{j}_{n} \phi \subset \mathcal{D}^{(n)}$. In computations, we work in local coordinates $\left(z, Z^{(n)}\right)$ on $\mathcal{D}^{(n)}$ provided by a system of source coordinates $z=\left(z^{1}, \ldots, z^{m}\right)$ on $M$, target coordinates $Z=\left(Z^{1}, \ldots, Z^{m}\right)$ also on $M$, and associated jet coordinates $Z_{B}^{a}$ representing the partial derivatives $\partial^{k} \phi^{a}(z) / \partial z^{b_{1}} \cdots \partial z^{b_{k}}$ of the local diffeomorphism $Z=\phi(z)$, with $1 \leq a, b_{1}, \ldots, b_{k} \leq m, 1 \leq k=\# A \leq$ $n$. Following Cartan, $[\mathbf{1 7}, \mathbf{1 8}]$, we will consistently use lower case letters, $z, x, u, \ldots$ for the source coordinates and the corresponding upper case letter $Z, X, U, \ldots$ for the target coordinates of local diffeomorphisms $Z=\phi(z)$. For $k \geq n$, let $\pi_{n}^{k}: \mathcal{D}^{(k)} \rightarrow \mathcal{D}^{(n)}$ denote the standard projection, so $\pi_{n}^{k}\left(z, Z^{(k)}\right)=\left(z, Z^{(n)}\right)$. 
The diffeomorphism jet bundle $\mathcal{D}^{(n)}$ forms a groupoid, $[\mathbf{4 8}, \mathbf{9 4}]$, with source map $\sigma\left(\left.\mathrm{j}_{n} \phi\right|_{z}\right)=z$ and target map $\tau\left(\left.\mathrm{j}_{n} \phi\right|_{z}\right)=\phi(z)=Z$. The groupoid multiplication is induced by composition of diffeomorphisms, and requires that, in any product of diffeomorphism jets, the source of the left multiplicand match the target of the right.

Example 2.2. Consider the simplest case $M=\mathbb{R}$ with coordinate $x$. Local coordinates on $\mathcal{D}^{(n)}(\mathbb{R})$ are denoted $\left(x, X, X_{x}, X_{x x}, \ldots, X_{n}\right)$, where $X_{k}$ corresponds to the $k^{\text {th }}$ derivative of the diffeomorphism $X=\phi(x)$ at the source point $x$. The groupoid multiplication corresponds to composition of Taylor polynomials/series, so that

$$
\left(X, \mathbf{X}, \mathbf{X}_{X}, \mathbf{X}_{X X}, \ldots\right) \cdot\left(x, X, X_{x}, X_{x x}, \ldots\right)=\left(x, \mathbf{X}, \mathbf{X}_{X} X_{x}, \mathbf{X}_{X} X_{x x}+\mathbf{X}_{X X} X_{x}^{2}, \ldots\right),
$$

which is only defined when the source coordinate $X$ of the left hand jet matches the target coordinate of the right hand jet. Higher-order terms can be expressed in terms of Bell polynomials via the general Fàa-di-Bruno formula, $[\mathbf{6 3}, \mathbf{8 0}]$.

Given a pseudo-group $\mathcal{G} \subset \mathcal{D}$, let $\mathcal{G}^{(n)} \subset \mathcal{D}^{(n)}$ denote the corresponding subgroupoid consisting of the $n^{\text {th }}$ order jets of its local diffeomorphisms. To avoid unresolved complications at singularities, we will impose the following blanket regularity condition throughout.

Definition 2.3. A pseudo-group $\mathcal{G} \subset \mathcal{D}$ is called regular of order $n^{\star} \geq 1$ if, for all finite $n \geq n^{\star}$, the pseudo-group jets form an embedded subbundle $\mathcal{G}^{(n)} \subset \overline{\mathcal{D}}^{(n)}$ and the projection $\pi_{n}^{n+1}: \mathcal{G}^{(n+1)} \rightarrow \mathcal{G}^{(n)}$ is a fibration.

The fiber dimension $r_{n}=\left.\operatorname{dim} \mathcal{G}^{(n)}\right|_{z}$, for $z \in M$, quantifies the number of independent pseudo-group parameters of order $\leq n$. If $r_{n}=r$ for all $n \gg 0$, then $\mathcal{G}$ is of finite type, and represents the (local) action of an $r$-dimensional Lie group $G$. In this case, the bundles $\mathcal{G}^{(n)} \rightarrow M$ for $n \gg 0$ are principal bundles with structure group $G$.

Definition 2.4. An analytic pseudo-group $\mathcal{G} \subset \mathcal{D}$ is called a Lie pseudo-group if $\mathcal{G}$ is regular of order $n^{\star} \geq 1$ and, moreover, every local diffeomorphism $\phi \in \mathcal{D}$ satisfying $\mathrm{j}_{n^{\star}} \phi \subset \mathcal{G}^{\left(n^{\star}\right)}$ belongs to the pseudo-group: $\phi \in \mathcal{G}$.

In local coordinates, the pseudo-group jet subbundle $\mathcal{G}^{\left(n^{\star}\right)} \subset \mathcal{D}^{\left(n^{\star}\right)}$ is characterized by a system of $n^{\star}$-th order partial differential equations

$$
F^{\left(n^{\star}\right)}\left(z, Z^{\left(n^{\star}\right)}\right)=0,
$$

known as the determining system for the pseudo-group. The Lie condition of Definition 2.4 requires that every local solution $Z=\phi(z)$ to the determining system is a pseudo-group transformations. (Global solutions need not be one-to-one, and so may be excluded.) In most treatments of the subject, an additional integrability or involutivity requirement, $[\mathbf{1 1}, \mathbf{6 5}, \mathbf{8 3}]$, is imposed on the determining system (2.1). However, in the analytic category, involutivity is, in fact, a direct consequence of regularity, [35].

Theorem 2.5. The determining equations of a regular analytic Lie pseudo-group of order $n^{\star}$ are involutive at some order $n \geq n^{\star}$. 
Proof: Recall that the $k^{\text {th }}$ prolongation of a system of differential equations is defined as the system obtained by appending all (total) derivatives of the original equations of orders $\leq k$. We write $\operatorname{pr}^{(k)}$ for the prolongation operation. By regularity and analyticity, the Cartan-Kuranishi Theorem, $[\mathbf{1 1}, \mathbf{1 5}, \mathbf{4 2}, \mathbf{8 3}]$, implies that some projection/prolongation $\widetilde{\mathcal{G}}^{(n)} \equiv \pi_{n}^{k} \operatorname{pr}^{\left(k-n^{\star}\right)} \mathcal{G}^{\left(n^{\star}\right)} \subset \mathcal{D}^{(n)}, k \geq n \geq n^{\star}$, is involutive. We claim that $\widetilde{\mathcal{G}}^{(n)}=\mathcal{G}^{(n)}$. Indeed, any solution $\phi \in \mathcal{G}$ to the determining equations $\mathcal{G}^{\left(n^{\star}\right)}$ is automatically a solution to any prolongation and projection thereof, and hence satisfies the involutive system $\widetilde{\mathcal{G}}^{(n)}$. But $\mathcal{G}^{(n)}$ is, by definition, the set of all solution jets of order $n$, and thus $\mathcal{G}^{(n)} \subset \widetilde{\mathcal{G}}^{(n)}$. On the other hand, any solution to $\widetilde{\mathcal{G}}^{(n)}$ is necessarily a solution to the original system $\mathcal{G}^{\left(n^{\star}\right)}$ and thus, since $\mathcal{G}$ is, by assumption, a Lie pseudo-group, an element of $\mathcal{G}$. We conclude that $\widetilde{\mathcal{G}}^{(n)} \subset \mathcal{G}^{(n)}$, proving their equality.

Q.E.D.

In the smooth category, there are no comparable existence theorems for involutive systems of partial differential equations, and so involutivity must be included in the definition of a Lie pseudo-group. Indeed, it is conceivable that a $\mathrm{C}^{\infty}$ system of partial differential equations be local solvable and yet not formally integrable due to some "hidden integrability condition" satisfied by its higher order jets that cannot be deduced by prolongation. In more detail, it may be possible that a smooth pseudo-group $\mathcal{G}$ satisfy the conditions of Definition 2.4, and yet, for some $n>n^{\star}$, the $n^{\text {th }}$ order pseudo-group jets form a strict subbundle of the prolonged determining system: $\mathcal{G}^{(n)} \subsetneq \mathrm{pr}^{\left(n-n^{\star}\right)} \mathcal{G}^{\left(n^{\star}\right)}$, meaning that some of the differential equations required to specify $\mathcal{G}^{(n)}$ for some $n>n^{\star}$ do not result from differentiating its determining system $\mathcal{G}^{\left(n^{\star}\right)}$. Finding such an example — or, alternatively, proving that such does not exist - is a challenging problem.

While the Lie condition of Definition 2.4 imposes a technical restriction on the types of pseudo-groups to be considered, in a certain sense it is automatic. Namely, any regular pseudo-group $\mathcal{G}$ has a canonical Lie completion $\overline{\mathcal{G}} \supseteq \mathcal{G}$ - namely, the set of all analytic solutions to its determining system $\mathcal{G}^{\left(n^{\star}\right)}=\overline{\mathcal{G}}^{\left(n^{\star}\right)}$. Moreover, the original pseudo-group and its Lie completion are indistinguishable as far as their local geometry, e.g., differential invariants, the invariant variational bicomplex, etc., is concerned, [35].

Theorem 2.6. Any regular non-Lie pseudo-group can be canonically completed to a Lie pseudo-group with the same differential invariants and invariant differential forms.

Example 2.7. Let the pseudo-group $\mathcal{G}_{d}$ be given by the diagonal action $X=f(x)$, $Y=f(y)$, of $f \in \mathcal{D}(\mathbb{R})$ on the open submanifold $M=\{x \neq y\} \subset \mathbb{R}^{2}$. In terms of the local coordinates $\left(x, y, X, Y, X_{x}, X_{y}, Y_{x}, Y_{y}\right)$ on $\mathcal{D}^{(1)}$, its first order jets $\mathcal{G}_{d}^{(1)}$ are subject to the determining system $X_{y}=Y_{x}=0, X_{x}, Y_{y} \neq 0$; higher order subbundles $\mathcal{G}_{d}^{(n)} \subset \mathcal{D}^{(n)}$ are obtained by prolongation (differentiation). Its Lie completion $\overline{\mathcal{G}}_{d} \supsetneq \mathcal{G}_{d}$ is the Lie pseudogroup formed by the general solution to the determining system - namely, $X=f(x)$, $Y=g(y)$, where $f, g \in \mathcal{D}(\mathbb{R})$ are independent local diffeomorphisms.

\section{Maurer-Cartan Forms and Structure Equations.}

Let us turn to the basic structure theory of Lie pseudo-groups. Cartan's approach, $[\mathbf{1 7}, \mathbf{1 8}, \mathbf{3 6}, \mathbf{8 8}]$, relies on the prolongation of certain exterior differential systems, $[\mathbf{1 1}]$. As 
such, it suffers from several drawbacks. First, in the intransitive case, Cartan's algorithm requires an adapted coordinate system that involves the pseudo-group invariants, whose calculation may not be easy. Secondly, the geometric interpretation of the differential forms resulting from his intricate prolongation procedure is not so evident. Furthermore, when applied to finite-dimensional intransitive actions, the resulting structure equations are not the same as the standard Maurer-Cartan equations for the Lie group; indeed, elementary abelian Lie group actions may end up with nonzero structure coefficients. Moreover, isomorphic pseudo-group actions may admit non-isomorphic Cartan structure equations. We refer the reader to $[\mathbf{6 0}, \mathbf{7 6}, \mathbf{9 1}, \mathbf{9 2}]$ for further details.

In contrast, our new approach to the structure theory is directly inspired by the classical invariant Maurer-Cartan forms on a finite-dimensional Lie group. To adapt to general pseudo-groups, the Maurer-Cartan forms will be regarded as living on the principal bundles $\mathcal{G}^{(n)} \rightarrow M$ formed by the group transformation jets of sufficiently high order. Generalizing this construction, the Maurer-Cartan forms of a Lie pseudo-group are identified as right-invariant ${ }^{\dagger}$ one-forms on the pseudo-group jet bundles $\mathcal{G}^{(n)}$. The invariant forms can, in fact, be explicitly constructed, and the resulting structure equations immediately established, using only linear algebra, from the pseudo-group's infinitesimal generators. This direct approach successfully avoids all of the aforementioned difficulties associated with Cartan's method. One can work in arbitrary local coordinates; the geometrical interpretation of the resulting Maurer-Cartan forms is immediate; the structure equations are intrinsically dual to the commutator equations for the infinitesimal generators, and thus coincide with the standard Lie group structure equations when the pseudo-group is of finite type; finally, isomorphic pseudo-groups necessarily have isomorphic structure equations.

The main tool is the powerful variational bicomplex structure possessed by the differential forms on jet bundles, $[\mathbf{1}, \mathbf{2}, \mathbf{9 0}]$. The cotangent space on the infinite diffeomorphism jet bundle $\mathcal{D}^{(\infty)} \subset \mathrm{J}^{\infty}(M, M)$ naturally splits ${ }^{\ddagger}$ into horizontal and vertical (contact) components. In terms of local coordinates $z^{a}, Z_{B}^{a}$, the horizontal subbundle is spanned by the one-forms $d z^{a}=d_{M} z^{a}, a=1, \ldots, m$, while the vertical subbundle is spanned by the basic contact one-forms

$$
\Upsilon_{B}^{a}=d_{G} Z_{B}^{a}=d Z_{B}^{a}-\sum_{c=1}^{m} Z_{B, c}^{a} d z^{c}, \quad a=1, \ldots, m, \quad \# B \geq 0,
$$

distinguished by their vanishing on all diffeomorphism jets $\mathrm{j}_{\infty} \phi \subset \mathcal{D}^{(\infty)},[\mathbf{6 5}, \mathbf{7 2}]$. We use $d=d_{M}+d_{G}$ to denote the induced splitting of the differential. In coordinates,

$$
d_{M} F=\sum_{a=1}^{m}\left(\mathbb{D}_{z^{a}} F\right) d z^{a}, \quad d_{G} F=\sum_{a=1}^{m} \sum_{\# B \geq 0} \frac{\partial F}{\partial Z_{B}^{a}} \Upsilon_{B}^{a},
$$

$\dagger$ As always, one needs to make a choice between right and left invariance. For our purposes, the right version is slightly more convenient.

$\ddagger$ The splitting only occurs at infinite order. However, computations always take place on finite order jet bundles. 
for any differential function $F\left(z, Z^{(n)}\right)$, with

$$
\mathbb{D}_{z^{a}}=\frac{\partial}{\partial z^{a}}+\sum_{c=1}^{m} \sum_{\# B \geq 0} Z_{B, a}^{c} \frac{\partial}{\partial Z_{B}^{c}}, \quad a=1, \ldots, m
$$

denoting the usual total derivative operators.

Composition of local diffeomorphisms induces an action of the diffeomorphism pseudogroup $\mathcal{D}$ on its jet groupoids $\mathcal{D}^{(n)}$, namely, $\mathrm{R}_{\phi}\left(\left.\mathrm{j}_{n} \psi\right|_{z}\right)=\left.\mathrm{j}_{n}\left(\psi \circ \phi^{-1}\right)\right|_{\phi(z)}$ for $\phi \in \mathcal{D}$. A differential form $\mu$ on $\mathcal{D}^{(n)}$ is right-invariant if $\mathrm{R}_{\phi}^{*} \mu=\mu$, where defined, for every $\phi \in \mathcal{D}$. This action preserves the bicomplex splitting, and so if $\mu$ is any right-invariant form, so are $d_{M} \mu$ and $d_{G} \mu$. In particular, the target coordinate functions $Z^{a}: \mathcal{D}^{(0)} \rightarrow \mathbb{R}$ are obviously right-invariant. Therefore, their horizontal differentials

$$
\sigma^{a}=d_{M} Z^{a}=\sum_{b=1}^{m} Z_{b}^{a} d z^{b}, \quad a=1, \ldots, m
$$

form a right-invariant horizontal coframe, while their vertical differentials

$$
\mu^{a}=d_{G} Z^{a}=d Z^{a}-\sum_{b=1}^{m} Z_{b}^{a} d z^{b}, \quad a=1, \ldots, m,
$$

are the zero ${ }^{\text {th }}$ order contact forms, which are thus also right-invariant. Let $\mathbb{D}_{Z^{1}}, \ldots, \mathbb{D}_{Z^{m}}$ be the total derivative operators dual to the horizontal coframe (3.4), satisfying

$$
d_{M} F=\sum_{a=1}^{m}\left(\mathbb{D}_{Z^{a}} F\right) d Z^{a}
$$

for any differential function $F\left(z, Z^{(n)}\right)$. Lie differentiation with respect to $\mathbb{D}_{Z^{a}}$ preserves right-invariance, and hence

$$
\mu_{B}^{a}=\mathbb{D}_{Z}^{B} \mu^{a}, \quad \text { where } \quad \mathbb{D}_{Z}^{B}=\mathbb{D}_{Z^{b_{1}}} \cdots \mathbb{D}_{Z^{b_{k}}}, \quad a=1, \ldots, m, \quad k=\# B \geq 0,
$$

form a basis for the right-invariant contact forms, which are to be viewed as the MaurerCartan forms for the diffeomorphism pseudo-group.

Example 3.1. When $M=\mathbb{R}$, using the coordinate notation of Example 2.2, the ordinary contact forms are

$$
\Upsilon=d X-X_{x} d x, \quad \Upsilon_{x}=d X_{x}-X_{x x} d x, \quad \Upsilon_{x x}=d X_{x x}-X_{x x x} d x
$$

and so on. Starting with the target coordinate $X$, the basic right-invariant horizontal form is $\sigma=d_{M} X=X_{x} d x$, with dual invariant differentiation

$$
\mathbb{D}_{X}=X_{x} \mathbb{D}_{x}=X_{x}\left(\frac{\partial}{\partial x}+X_{x} \frac{\partial}{\partial X}+X_{x x} \frac{\partial}{\partial X_{x}}+X_{x x x} \frac{\partial}{\partial X_{x x}}+\cdots\right) \text {. }
$$


Starting with $\mu=d_{G} X=\Upsilon$, the right-invariant contact forms are $\mu_{n}=\mathbb{D}_{X}^{n} \mu$ for $n=$ $0,1,2, \ldots ;$ explicitly,

$$
\mu_{0}=\Upsilon, \quad \mu_{1}=\mathbb{D}_{X} \mu=\frac{\Upsilon_{x}}{X_{x}}, \quad \mu_{2}=\mathbb{D}_{X}^{2} \mu=\frac{X_{x} \Upsilon_{x x}-X_{x x} \Upsilon_{x}}{X_{x}^{3}}, \quad \ldots .
$$

These are to be regarded as the Maurer-Cartan forms for the diffeomorphism pseudo-group $\mathcal{D}(\mathbb{R})$.

The structure equations for the diffeomorphism groupoid $\mathcal{D}^{(\infty)}$ express the differentials $d \sigma^{a}, d \mu_{B}^{a}$ of the Maurer-Cartan forms as linear combinations of wedge products of MaurerCartan forms. They are most concisely formulated as follows, [72]. Let $\mu \llbracket H \rrbracket$ denote the column vector whose components are the invariant contact form-valued formal power series

$$
\mu^{a} \llbracket H \rrbracket=\sum_{\# B \geq 0} \frac{1}{B !} \mu_{B}^{a} H^{B}, \quad a=1, \ldots, m,
$$

depending on the formal parameters $H=\left(H^{1}, \ldots, H^{m}\right)$. Further, let $d Z=\mu+\sigma=\mu \llbracket 0 \rrbracket+$ $\sigma$ denote column vectors of one-forms whose entries are $d Z^{a}=\mu^{a}+\sigma^{a}$ for $a=1, \ldots, m$.

Theorem 3.2. The structure equations for the diffeomorphism pseudo-group are obtained by equating individual coefficients in the formal power series identities

$$
d \mu \llbracket H \rrbracket=\nabla_{H} \mu \llbracket H \rrbracket \wedge(\mu \llbracket H \rrbracket-d Z), \quad d \sigma=-d \mu \llbracket 0 \rrbracket=\nabla_{H} \mu \llbracket 0 \rrbracket \wedge \sigma .
$$

Here $\nabla_{H} \mu \llbracket H \rrbracket=\left(\frac{\partial \mu^{a}}{\partial H^{b}} \llbracket H \rrbracket\right)$ denotes the $m \times m$ formal power series Jacobian matrix.

Example 3.3. Continuing Example 3.1, when $M=\mathbb{R}$, the Maurer-Cartan form series is

$$
\mu \llbracket H \rrbracket=\mu_{0}+\mu_{1} H+\frac{1}{2} \mu_{2} H^{2}+\frac{1}{6} \mu_{3} H^{3}+\cdots .
$$

The diffeomorphism structure equations (3.12) take the form

$$
d \sigma=\mu_{1} \wedge \sigma, \quad d \mu \llbracket H \rrbracket=\mu^{\prime} \llbracket H \rrbracket \wedge(\mu \llbracket H \rrbracket-d Z),
$$

where

$$
\mu^{\prime} \llbracket H \rrbracket=\mu_{1}+\mu_{2} H+\frac{1}{2} \mu_{3} H^{2}+\cdots
$$

is the formal derivative of the Maurer-Cartan form series $\mu \llbracket H \rrbracket$ with respect to $H$, while

$$
\mu \llbracket H \rrbracket-d Z=-\sigma+\mu_{1} H+\frac{1}{2} \mu_{2} H^{2}+\cdots .
$$

Equating the coefficients of the various powers of $H$ in the second structure equation (3.13), we recover Cartan's formulae, [17; eq. (48)], for the structure equations for the diffeomorphism pseudo-group $\mathcal{D}(R)$ :

$$
d \sigma=\mu_{1} \wedge \sigma, \quad d \mu_{n}=\sigma \wedge \mu_{n+1}-\sum_{j=1}^{[(n+1) / 2]} \frac{n-2 j+1}{n+1}\left(\begin{array}{c}
n+1 \\
j
\end{array}\right) \mu_{j} \wedge \mu_{n+1-j}, \quad n \geq 0 .
$$


As Lie discovered, the key to analyzing pseudo-group actions is to work infinitesimally. Let $\mathfrak{g}$ denote the Lie algebra ${ }^{\dagger}$ of infinitesimal generators of the pseudo-group, i.e., the set of locally defined vector fields (3.14) whose flows belong to $\mathcal{G}$. In local coordinates, a vector field on $M$ takes the form

$$
\mathbf{v}=\sum_{a=1}^{m} \zeta^{a}(z) \frac{\partial}{\partial z^{a}} .
$$

Let $\mathrm{J}^{n} T M$, for $0 \leq n \leq \infty$, denote the tangent $n$-jet bundle. Local coordinates on $\mathrm{J}^{n} T M$ are indicated by

$$
\left(z, \zeta^{(n)}\right)=\left(\ldots z^{a} \ldots \zeta_{B}^{a} \ldots\right), \quad a=1, \ldots, m, \quad 0 \leq \# B \leq n,
$$

where $\zeta_{B}^{a}$ represents the partial derivative $\partial^{B} \zeta^{a} / \partial z^{B}$. For each $n \geq 0$, the subbundle $\mathrm{j}_{n} \mathfrak{g} \subset \mathrm{J}^{n} T M$ is prescribed by a system of linear partial differential equations

$$
L^{(n)}\left(z, \zeta^{(n)}\right)=0 .
$$

These can be obtained by linearizing the nonlinear determining system (2.1) at the identity jet, and are hence known as the linearized or infinitesimal determining system for the pseudo-group. If $\mathcal{G}$ arises as the symmetry group of a system of differential equations, then the linearized determining system (3.15) is the involutive completion of the usual infinitesimal determining equations obtained via Lie's algorithm; see Section 4 below for details.

As with finite-dimensional Lie groups, the structure of a pseudo-group is described by its invariant Maurer-Cartan forms. A complete system of right-invariant one-forms on $\mathcal{G}^{(\infty)} \subset \mathcal{D}^{(\infty)}$ is obtained by restricting (or pulling back) the diffeomorphism MaurerCartan forms $(3.4,7)$. We use the same notation $\sigma^{a}, \mu_{B}^{a}$ for the restricted forms, which are, of course, no longer linearly independent, but are subject to certain constraints prescribed by the pseudo-group. Remarkably, these constraints can be explicitly characterized by an invariant version of the linearized determining equations.

Theorem 3.4. For each $n \geq 0$, the homogeneous linear algebraic system

$$
L^{(n)}\left(Z, \mu^{(n)}\right)=0
$$

that is obtained from the infinitesimal determining equations (3.15) by formally replacing the source coordinates $z^{a}$ by the corresponding target coordinates $Z^{a}$, and the vector field jet coordinates $\zeta_{B}^{a}$ by the corresponding Maurer-Cartan forms $\mu_{B}^{a}$, serves to define the complete set of dependencies among the Maurer-Cartan forms $\mu^{(n)}$ when restricted to the pseudo-group jet subbundle $\mathcal{G}^{(\infty)} \subset \mathcal{D}^{(\infty)}$.

Corollary 3.5. The structure equations for a pseudo-group $\mathcal{G}$ are given by restricting the diffeomorphism structure equations (3.12) to the solution space of (3.16).

$\dagger$ Here, we are using the term "Lie algebra" somewhat loosely, since, technically, the infinitesimal generators may only be locally defined, and so their Lie brackets only make sense on their common domains of definition. 
Example 3.6. Let $M=\{(x, u) \mid u \neq 0\} \subset \mathbb{R}^{2}$. Consider the transitive Lie pseudogroup $\mathcal{G} \subset \mathcal{D}(M)$ consisting of (local) diffeomorphisms of the form

$$
X=f(x), \quad U=\frac{u}{f^{\prime}(x)},
$$

where $f(x) \in \mathcal{D}(\mathbb{R})$ is an arbitrary local diffeomorphism. The pseudo-group jets are obtained by differentiation of the target coordinates $X, U$ with respect to the source coordinates $x, u$; to first order,

$$
X=f, \quad U=\frac{u}{f_{x}}, \quad X_{x}=f_{x}, \quad X_{u}=0, \quad U_{x}=-\frac{u f_{x x}}{f_{x}^{2}}, \quad U_{u}=\frac{1}{f_{x}} .
$$

The determining system is obtained by implicitization, whence

$$
U_{u}=\frac{U}{u}, \quad U X_{x}=u, \quad X_{u}=0 .
$$

This system is involutive, and so the higher order determining systems can be obtained by repeated differentiation of (3.19).

The infinitesimal generators of (3.17) are all vector fields of the form

$$
\mathbf{v}=\xi(x, u) \frac{\partial}{\partial x}+\varphi(x, u) \frac{\partial}{\partial u}=a(x) \frac{\partial}{\partial x}-a^{\prime}(x) u \frac{\partial}{\partial u},
$$

where $a(x)$ is an arbitrary analytic function. The coefficients $\xi(x, u)=a(x), \varphi(x, u)=$ $-u a^{\prime}(x)$, form the general solution to the first order infinitesimal determining system

$$
\varphi_{u}=\frac{\varphi}{u}=-\xi_{x}, \quad \xi_{u}=0
$$

which is obtained by linearizing the determining system (3.19) at the identity jet.

The Maurer-Cartan forms are obtained by restricting the diffeomorphism MaurerCartan forms to the subbundle defined by the pseudo-group determining equations (3.19). The zeroth and first order Maurer-Cartan forms are

$$
\begin{array}{ll}
\sigma=X_{x} d x+X_{u} d u=f_{x} d x, & \tau=U_{x} d x+U_{u} d u=\frac{-u f_{x x} d x+f_{x} d u}{f_{x}^{2}} \\
\mu=d X-X_{x} d x-X_{u} d u=\Phi, & \nu=d U-U_{x} d x-U_{u} d u=-\frac{u \Phi_{x}}{f_{x}^{2}} \\
\mu_{X}=\frac{\Phi_{x}}{f_{x}}, \quad \nu_{U}=-\frac{\Phi_{x}}{f_{x}}, & \mu_{U}=0, \quad \nu_{X}=\frac{u f_{x x} \Phi_{x}-u f_{x} \Phi_{x x}}{f_{x}^{4}}
\end{array}
$$

where

$$
\Phi=d f-f_{x} d x, \quad \Phi_{x}=d f_{x}-f_{x x} d x,, \quad \Phi_{x x}=d f_{x x}-f_{x x x} d x, \quad \ldots,
$$

are contact forms in the pseudo-group parameters. Applying the replacement rules of Theorem 3.4 to the linearized determining equations (3.21), the Maurer-Cartan forms (3.22) are constrained by the linear algebraic constraints

$$
\nu_{U}=\frac{\nu}{U}=-\mu_{X}, \quad \quad \mu_{U}=0
$$


Higher-order constraints can be produced by formal prolongation, i.e., applying the replacement rules to the derivatives of (3.23). The remaining independent one-forms $\sigma, \tau, \mu$, $\nu, \nu_{X}, \nu_{X X}, \nu_{X X X}, \ldots$ form a right-invariant coframe on $\mathcal{G}^{(\infty)}$. As in Corollary 3.5 , the pseudo-group structure equations

$$
\begin{array}{ll}
d \sigma=-d \mu=-\frac{\nu \wedge \sigma}{U}, & d \mu_{X}=\frac{\nu_{X} \wedge \sigma}{U}, \\
d \tau=-d \nu=\nu_{X} \wedge \sigma+\frac{\nu \wedge \tau}{U}, & d \nu_{X}=-\nu_{X X} \wedge \sigma-\frac{\nu_{X} \wedge(\tau+2 \nu)}{U},
\end{array}
$$

and so on, are obtained by restriction of the diffeomorphism structure equations to (3.23).

\section{Symmetries of Differential Equations.}

One of the most common arenas in which Lie pseudo-groups arise is, not surprisingly, in Lie's theory of symmetry group of differential equations. In this section, we review the basic ideas. Applications of symmetry groups to the construction of explicit solutions, integration of ordinary differential equations, construction of explicit solutions to partial differential equations, determination of conservation laws, etc., can be found, for instance, in $[13,64,65]$.

As before, let $M$ be an analytic, $m$-dimensional manifold. Fix $0<p<m$. For each integer $0 \leq n \leq \infty$, let $\mathrm{J}^{n}=\mathrm{J}^{n}(M, p)$ denote the $n^{\text {th }}$ order submanifold jet bundle, which is prescribed by the equivalence relation of $n^{\text {th }}$ order contact of $p$-dimensional submanifolds $N \subset M$. For $k \geq n$, we use $\pi_{n}^{k}: \mathrm{J}^{k} \rightarrow \mathrm{J}^{n}$ to denote the natural projection. Introducing local coordinates $z=(x, u)$ on $M$, we consider the first $p$ components $x=\left(x^{1}, \ldots, x^{p}\right)$ as independent variables, and the latter $q=m-p$ components $u=\left(u^{1}, \ldots, u^{q}\right)$ as dependent variables, and we identify graphs $u=f(x)$ as submanifolds. The induced coordinates on $\mathrm{J}^{n}$ are denoted by $z^{(n)}=\left(x, u^{(n)}\right)$, with components $x^{i}$ and $u_{J}^{\alpha}=\partial^{J} u^{\alpha} / \partial x^{J}$ representing the derivatives of the $u$ 's with respect to to the $x$ 's of orders $0 \leq \# J \leq n$. A real-valued function $F\left(x, u^{(n)}\right)$, defined on an open subset of $\mathrm{J}^{n}$, is known as a differential function.

A system of $n^{\text {th }}$ order (partial) differential equations in $p$ independent variables and $q$ dependent variables forms a subvariety $\mathcal{S} \subset \mathrm{J}^{n}$. A (classical) solution to the system is a $p$-dimensional submanifold $N \subset M$ whose jet lies entirely in the subvariety: $\mathrm{j}_{n} N \subset \mathcal{S}$. We assume that the system is regular, meaning that it forms a submanifold, and hence $\mathcal{S} \subset \mathrm{J}^{n}$ is prescribed by the vanishing of a set of differential functions:

$$
\Delta_{\nu}\left(x, u^{(n)}\right)=0, \quad \nu=1, \ldots, l,
$$

whose Jacobian matrix has maximal rank at all jets $z^{(n)}=\left(x, u^{(n)}\right) \in \mathcal{S}$. We also assume the system is locally solvable, $[\mathbf{6 4}]$, meaning that there exists at least one solution passing through every jet in $\mathcal{S}$.

In general, by a symmetry of the system (4.1) we mean a transformation which takes solutions to solutions. Following Lie, we work infinitesimally. The prolongation of a vector field on $M$ to the submanifold jet bundle $\mathrm{J}^{n}$ has the form

$$
\mathbf{v}^{(n)}=\sum_{i=1}^{p} \xi^{i}(x, u) \frac{\partial}{\partial x^{i}}+\sum_{\alpha=1}^{q} \sum_{\# J \leq n} \varphi_{J}^{\alpha}\left(x, u^{(n)}\right) \frac{\partial}{\partial u_{J}^{\alpha}},
$$


whose coefficients are given by the prolongation formula

$$
\varphi_{J}^{\alpha}\left(x, u^{(n)}\right)=\mathrm{D}_{J}\left(\varphi^{\alpha}(x, u)-\sum_{i=1}^{p} \xi^{i}(x, u) u_{i}^{\alpha}\right)+\sum_{i=1}^{p} \xi^{i}(x, u) u_{J, i}^{\alpha},
$$

first stated in this form in $[\mathbf{6 3}]$. Here

$$
\mathrm{D}_{i}=\frac{\partial}{\partial x^{i}}+\sum_{\alpha=1}^{q} \sum_{\# J \geq 0} u_{J, i}^{\alpha} \frac{\partial}{\partial u_{J}^{\alpha}}, \quad i=1, \ldots, p,
$$

are the total derivatives on $\mathrm{J}^{n}$, while $\mathrm{D}_{J}=\mathrm{D}_{j_{1}} \cdot \ldots \cdot \mathrm{D}_{j_{k}}$ for $k=\# J \geq 0$.

Theorem 4.1. A connected Lie pseudo-group $\mathcal{G}$ forms a symmetry group of the regular system of differential equations $\mathcal{S} \subset \mathrm{J}^{n}$ if and only if its prolonged infinitesimal generators are everywhere tangent to $\mathcal{S}$.

In local coordinates, the tangency condition leads to the classical infinitesimal symmetry criterion

$$
\mathbf{v}^{(n)}\left(\Delta_{\nu}\right)=0, \quad \nu=1, \ldots, r, \quad \text { whenever } \quad \Delta=0 .
$$

This forms a large over-determined linear system of partial differential equations for the coefficients $\xi^{i}, \varphi^{\alpha}$ of the infinitesimal symmetry generator $\mathbf{v}$, known as the infinitesimal determining equations for the symmetry pseudo-group of the original system.

Example 4.2. The celebrated Korteweg-deVries (KdV) equation, [64], is

$$
u_{t}+u_{x x x}+u u_{x}=0 .
$$

According to (4.5), a vector field

$$
\mathbf{v}=\tau(t, x, u) \frac{\partial}{\partial t}+\xi(t, x, u) \frac{\partial}{\partial x}+\varphi(t, x, u) \frac{\partial}{\partial u}
$$

forms an infinitesimal symmetry of the $\mathrm{KdV}$ equation if and only if

$$
\mathbf{v}^{(3)}\left(u_{t}+u_{x x x}+u u_{x}\right)=\varphi^{t}+\varphi^{x x x}+u \varphi^{x}+u_{x} \varphi=0 \quad \text { whenever } \quad u_{t}+u_{x x x}+u u_{x}=0 .
$$

Here $\varphi^{t}, \varphi^{x}, \varphi^{x x x}$ are, respectively, the coefficients of $\partial_{u_{x}}, \partial_{u_{x}}, \partial_{u_{x x x}}$ in the prolongation of (4.7). Substituting the prolongation formula (4.2), and equating the coefficients of the independent derivative monomials to zero, leads to the infinitesimal determining equations

$$
\tau_{x}=\tau_{u}=\xi_{u}=\varphi_{t}=\varphi_{x}=0, \quad \varphi=\xi_{t}-\frac{2}{3} u \tau_{t}, \quad \varphi_{u}=-\frac{2}{3} \tau_{t}=-2 \xi_{x} .
$$

Differentiation implies that all the second and higher order derivatives vanish. The general solution

$$
\tau=c_{1}+3 c_{4} t, \quad \xi=c_{2}+c_{3} t+c_{4} x, \quad \varphi=c_{3}-2 c_{4} u,
$$

defines the four-dimensional KdV symmetry algebra, spanned by

$$
\mathbf{v}_{1}=\partial_{t}, \quad \mathbf{v}_{2}=\partial_{x}, \quad \mathbf{v}_{3}=t \partial_{x}+\partial_{u}, \quad \mathbf{v}_{4}=3 t \partial_{t}+x \partial_{x}-2 u \partial_{u}
$$


The action of the KdV symmetry group $G_{K d V}$ can be obtained by composing the flows of the symmetry algebra basis:

$$
\begin{aligned}
(T, X, U) & =\exp \left(\lambda_{4} \mathbf{v}_{4}\right) \circ \exp \left(\lambda_{3} \mathbf{v}_{3}\right) \circ \exp \left(\lambda_{2} \mathbf{v}_{2}\right) \circ \exp \left(\lambda_{1} \mathbf{v}_{1}\right)(t, x, u) \\
& =\left(e^{3 \lambda_{4}}\left(t+\lambda_{1}\right), e^{\lambda_{4}}\left(\lambda_{3} t+x+\lambda_{1} \lambda_{3}+\lambda_{2}\right), e^{-2 \lambda_{4}}\left(u+\lambda_{3}\right)\right) .
\end{aligned}
$$

To obtain the structure equations, as in (3.16), we substitute $(t, x, u) \mapsto(T, X, U)$ and $(\tau, \xi, \varphi) \mapsto\left(\mu^{t}, \mu^{x}, \mu^{u}\right)$ into the infinitesimal determining equations (4.8), resulting in the following linear algebraic relations among the first order diffeomorphism Maurer-Cartan forms:

$$
\mu_{X}^{t}=\mu_{U}^{t}=\mu_{U}^{x}=\mu_{T}^{u}=\mu_{X}^{u}=0, \quad \mu^{u}=\mu_{T}^{x}-\frac{2}{3} U \mu_{T}^{t}=0, \quad \mu_{U}^{u}=-\frac{2}{3} \mu_{T}^{t}=-2 \mu_{X}^{x},
$$

while all the second and higher order forms are zero. Solving this homogeneous linear system, we find that there are precisely 4 independent invariant contact forms, namely $\mu^{t}, \mu^{x}, \mu^{u}, \mu_{T}^{t}$, which reflects the fact that $G_{K d V}$ is a four-dimensional Lie group. The structure equations of the complete invariant coframe are obtained by restricting the full diffeomorphism structure equations:

$$
\begin{gathered}
d \sigma^{t}=\mu^{4} \wedge \sigma^{t}, \quad d \sigma^{x}=\mu^{3} \wedge \sigma^{t}+\frac{2}{3} U \mu^{4} \wedge \sigma^{t}+\frac{1}{3} \mu^{4} \wedge \sigma^{x}, \quad d \sigma^{u}=-\frac{2}{3} \mu^{4} \wedge \sigma^{u}, \\
d \mu^{1}=-\mu^{4} \wedge \sigma^{t}, \quad d \mu^{2}=-\mu^{3} \wedge \sigma^{t}-\frac{2}{3} U \mu^{4} \wedge \sigma^{t}-\frac{1}{3} \mu^{4} \wedge \sigma^{x}, \quad d \mu^{3}=\frac{2}{3} \mu^{4} \wedge \sigma^{u}, \quad d \mu^{4}=0,
\end{gathered}
$$

where $\sigma^{t}, \sigma^{x}, \sigma^{u}$ are the invariant horizontal forms. This coframe lives on the principal bundle $G_{K d V}^{(1)}$. The classical Maurer-Cartan equations for $\mathfrak{g}_{K d V}^{*}$ are obtained by restricting to an individual target fiber, where $Z=(T, X, U)$ are fixed:

$$
\begin{array}{ll}
d \mu^{1}=-\mu^{1} \wedge \mu^{4}, & d \mu^{3}=\frac{2}{3} \mu^{3} \wedge \mu^{4}, \\
d \mu^{2}=-\mu^{1} \wedge \mu^{3}-\frac{2}{3} U \mu^{1} \wedge \mu^{4}-\frac{1}{3} \mu^{2} \wedge \mu^{4}, & d \mu^{4}=0 .
\end{array}
$$

Observe that the target coordinate $U$ appears in the structure equations (4.11), which indicates that the Maurer-Cartan basis for the fiber cotangent space varies from point to point. Of course, since we are dealing with a finite-dimensional Lie group action, one can make a $Z$ dependent change of Maurer-Cartan basis that results in structure equations that have constant structure coefficients. In contrast, such a change of basis is not always possible for infinite-dimensional Lie pseudo-groups, and there may well be essential invariants that cannot be eliminated from the structure equations. The appearance of such essential invariants is one of the key impediments to the existence of an abstract object that can represent the pseudo-group independently of the manifold upon which it acts. See $[\mathbf{1 7}, \mathbf{7 6}, \mathbf{9 1}, \mathbf{9 2}]$ for additional details.

\section{Moving Frames for Pseudo-Groups.}

We now turn to the construction, $[\mathbf{7 3}, \mathbf{7 4}]$, of moving frames for prolonged Lie pseudogroup actions on submanifold jet bundles. We begin by pulling back the pseudo-group jet bundle $\mathcal{G}^{(n)} \rightarrow M$ to the submanifold jet bundle via the projection $\pi_{0}^{n}: \mathrm{J}^{n} \rightarrow M$, thereby producing a bundle $\mathcal{H}^{(n)} \rightarrow \mathrm{J}^{n}$. Local coordinates on $\mathcal{H}^{(n)}$ are indicated by $\left(z^{(n)}, g^{(n)}\right)$, where the base coordinates $z^{(n)}=\left(x, u^{(n)}\right) \in \mathrm{J}^{n}$ represent submanifold jets, while the fiber 
coordinates $g^{(n)}$ serve to parametrize the pseudo-group jets. The bundle $\mathcal{H}^{(n)}$ also carries the structure of a groupoid, with source map $\sigma\left(z^{(n)}, g^{(n)}\right)=z^{(n)}$, while the target map $\tau\left(z^{(n)}, g^{(n)}\right)=g^{(n)} \cdot z^{(n)}$ is the prolonged action of a pseudo-group diffeomorphism with $n$ jet $g^{(n)}$ at the source point $z=\pi_{0}^{n}\left(z^{(n)}\right)$ of the submanifold jet $z^{(n)}$.

Definition 5.1. Given a regular Lie pseudo-group $\mathcal{G}$ acting on $M$, a moving frame of order $n$ is an equivariant local section $\rho: \mathrm{J}^{n} \rightarrow \mathcal{H}^{(n)}$.

Equivariance refers to the groupoid structure on $\mathcal{H}^{(n)}$, so that, for a right-equivariant moving frame, $\rho\left(g^{(n)} \cdot z^{(n)}\right)=\rho\left(z^{(n)}\right) \cdot\left(g^{(n)}\right)^{-1}$ when defined. As in the finite-dimensional theory, the existence of a moving frame requires that the prolonged pseudo-group action be free and regular. Regularity means that the pseudo-group orbits form a regular foliation. In the finite-dimensional Lie group version, freeness at a jet $z^{(n)}$ requires that its isotropy subgroup be trivial, i.e., $g^{(n)} \cdot z^{(n)}=z^{(n)}$ if and only if $g=e$ is the identity element. Local freeness requires only that the isotropy subgroup is discrete, and is equivalent to the orbit through $z^{(n)}$ having the same dimension as the group. But infinite-dimensional pseudo-groups acting on finite-dimensional spaces inevitably have nontrivial isotropy, and so the freeness condition must be suitably reformulated.

Definition 5.2. The jet isotropy subgroup of a submanifold jet $z^{(n)} \in \mathrm{J}^{n}$ is defined as $\mathcal{G}_{z^{(n)}}=\tau^{-1}\left\{z^{(n)}\right\} \cap \sigma^{-1}\left\{z^{(n)}\right\} \subset \mathcal{H}^{(n)}$. The pseudo-group is said to act freely at $z^{(n)}$ if $\mathcal{G}_{z^{(n)}}=\left\{\left(z^{(n)}, \mathbb{1}^{(n)}\right)\right\}$, i.e., the only order $n$ pseudo-group jet $g^{(n)}$ that fixes $z^{(n)}$ is that of the identity diffeomorphism. The pseudo-group acts locally freely at $z^{(n)}$ if $\mathcal{G}_{z^{(n)}}$ is discrete.

We note that, when specialized to a finite-dimensional Lie group action, the pseudogroup freeness Definition 5.2 is slightly more general than the usual requirement that the isotropy subgroup be trivial. A foundational result, proved in [75] - see also [74] for an alternative proof of the locally free version - is the persistence of freeness.

Theorem 5.3. For $n>0$, if the pseudo-group $\mathcal{G}$ acts (locally) freely at $z^{(n)}$ then it acts (locally) freely at any $z^{(k)} \in \mathrm{J}^{k}, k>n$, with $\pi_{n}^{k}\left(z^{(k)}\right)=z^{(n)}$.

We say that $\mathcal{G}$ acts eventually freely if, for some $n>0$, it acts freely on an open subset $\mathcal{V}^{n} \subset \mathrm{J}^{n}$, and hence on the open subsets $\mathcal{V}^{k}=\left(\pi_{n}^{k}\right)^{-1} \mathcal{V}^{n} \subset \mathrm{J}^{k}$ for any $k \geq n$. The minimal such $n$ is called the order of freeness, and denoted $n_{\star}$. The pseudo-group acts locally freely at $z^{(n)} \in \mathrm{J}^{n}$ if and only if the orbit through $z^{(n)}$ has dimension $r_{n}=\left.\operatorname{dim} \mathcal{G}^{(n)}\right|_{z}$, the fiber dimension of the pseudo-group jet groupoid at a point $z=\pi_{0}^{n}\left(z^{(n)}\right)$, which requires, at the very least, that

$$
r_{n}=\left.\operatorname{dim} \mathcal{G}^{(n)}\right|_{z} \leq \operatorname{dim} \mathrm{J}^{n}=p+(m-p)\left(\begin{array}{c}
p+n \\
p
\end{array}\right) .
$$

Thus, freeness serves to bound the number of pseudo-group parameters at each sufficiently high order, providing a simpler alternative to the Spencer cohomological growth conditions imposed in $[\mathbf{4 0 , 4 1}]$. Pseudo-groups having too large a fiber dimension $r_{n}$ (or, loosely, too many independent parameters), e.g., symplectomorphisms, [54], will, typically, act transitively on (a dense open subset of) $\mathrm{J}^{n}$, and thus possess no non-constant invariants. 
Extending our methods and results to non-free pseudo-group actions remains a significant open problem.

A real-valued function $I\left(z^{(n)}\right)$ defined on an open subset of the submanifold jet space is known as a differential invariant if it is constant on the prolonged pseudo-group orbits, and hence $I\left(g^{(n)} \cdot z^{(n)}\right)=I\left(z^{(n)}\right)$ for all $\left(z^{(n)}, g^{(n)}\right) \in \mathcal{H}^{(n)}$ such that both $z^{(n)}=\sigma\left(z^{(n)}, g^{(n)}\right)$ and $g^{(n)} \cdot z^{(n)}=\tau\left(z^{(n)}, g^{(n)}\right)$ lie in the domain of $I$. Clearly, any functional combination of differential invariants is a differential invariant (on the common domain of definition) and thus we speak, somewhat loosely, of the algebra of differential invariants associated with the action of the transformation group on submanifolds of a specified dimension. Since differential invariants may only be locally defined, we should technically work the category of sheaves of differential invariants, $[\mathbf{4 0 , 4 1 ]}$. However, for our local constructions, this extra level of abstraction only serves to obscure the main ideas, and so we will leave their sheaf-theoretic reformulation as a simple translational exercise for the cognoscenti.

As in the finite-dimensional version, [28], moving frames are constructed through a normalization procedure based on a choice of cross-section $\mathcal{K}^{n} \subset \mathrm{J}^{n}$ to the pseudo-group orbits, that is, a transversal submanifold of the complementary dimension. Assuming freeness, the associated (locally defined) moving frame section $\rho: \mathrm{J}^{n} \rightarrow \mathcal{H}^{(n)}$ is uniquely characterized by the condition that $\tau\left(\rho\left(z^{(n)}\right)\right) \in \mathcal{K}^{n}$. For the infinite-dimensional pseudogroups, a new cross-section and corresponding moving frame must be selected at each order above the order of freeness. We require compatibility of the cross-sections, in the sense that $\pi_{n}^{k}\left(\mathcal{K}^{k}\right)=\mathcal{K}^{n}$ for all $k \geq n \geq n_{\star}$, which implies compatibility of the resulting moving frames: $\pi_{n}^{k}\left(\rho\left(z^{(k)}\right)\right)=\rho\left(\pi_{n}^{k}\left(z^{(k)}\right)\right)$. We will simply refer to such a compatible sequence of moving frames as a moving frame.

Definition 5.4. The invariantization $I=\iota(F)$ of a differential function $F: \mathrm{J}^{n} \rightarrow \mathbb{R}$ is the unique differential invariant that agrees with $F$ on the cross-section: $I\left|\mathcal{K}^{n}=F\right| \mathcal{K}^{n}$.

In particular, invariantization preserves differential invariants: $\iota(I)=I$, and hence defines a morphism that projects the algebra of differential functions to the algebra of differential invariants. In coordinates, invariantization is implemented by first transforming according to the pseudo-group, and then replacing all the pseudo-group parameters by their moving frame formulae. In particular, invariantizing the jet coordinate functions yields the fundamental differential invariants

$$
\left(H, I^{(n)}\right)=\left(\ldots H^{i} \ldots I_{J}^{\alpha} \ldots\right)=\iota\left(x, u^{(n)}\right) .
$$

The combinations defining the cross-section $\mathcal{K}^{n}$ are constant, and called the phantom differential invariants, while the remaining basic differential invariants form a complete system of functionally independent differential invariants of order $\leq n$. With these in hand, the invariantization of a general function $F(z)$ is simply implemented by replacing each jet coordinate by the corresponding fundamental differential invariant:

$$
\iota\left[F\left(x, u^{(n)}\right)\right]=F\left(H, I^{(n)}\right) .
$$

In particular, this allows one to straightforwardly rewrite any other differential invariant in terms the basic invariants:

$$
J\left(x, u^{(n)}\right)=J\left(H, I^{(n)}\right) \quad \text { whenever } J \text { is a differential invariant, }
$$


an elementary but powerful result known as the Replacement Theorem.

To proceed further, we appeal to the variational bicomplex structure on the infinite order submanifold jet space, $[\mathbf{1}, \mathbf{2}, \mathbf{3 9}]$. Thus, the theory rests on the interactions two different variational bicomplexes - the first on the diffeomorphism jet space and the second on the submanifold jet space. The specification of independent and dependent variables on $M$ splits the differential one-forms on $\mathrm{J}^{\infty}=\mathrm{J}^{\infty}(M, p)$ into horizontal forms, spanned by $d x^{1}, \ldots, d x^{p}$, and contact forms, spanned by the basic contact forms

$$
\theta_{J}^{\alpha}=d u_{J}^{\alpha}-\sum_{i=1}^{p} u_{J, i}^{\alpha} d x^{i}, \quad \alpha=1, \ldots, q, \quad 0 \leq \# J .
$$

We let $\pi_{H}$ and $\pi_{V}$ be the projections onto horizontal and contact components, respectively. The differential on $\mathrm{J}^{\infty}$ splits into horizontal and vertical components, $d=d_{H}+d_{V}$, where $d_{H}=\pi_{H} \circ d, d_{V}=\pi_{V} \circ d$, satisfy $d_{H} \circ d_{H}=0=d_{V} \circ d_{V}$, while $d_{H} \circ d_{V}=-d_{V} \circ d_{H}$.

The invariantization process induced by a moving frame can also be applied to differential forms. Thus, given a differential form $\omega$, its invariantization $\iota(\omega)$ is the unique invariant differential form that agrees with $\omega$ on the cross-section. An invariantized contact form remains a contact form, while an invariantized horizontal form is, in general, a combination of horizontal and contact forms. The complete collection of invariantized differential forms forms the invariant variational bicomplex, [39].

Since this paper will concentrate on the differential invariants, we can safely ignore any contact forms. (On the other hand, they are required when dealing with invariant variational problems, $[\mathbf{3 9}]$, or submanifold flows, $[\mathbf{7 0}]$.) We will use the notation $\omega \equiv \varpi$ to indicate that two forms differ by a contact form. The horizontal components of the invariantized horizontal forms

$$
\omega^{i}=\pi_{H}\left[\iota\left(d x^{i}\right)\right] \equiv \iota\left(d x^{i}\right), \quad i=1, \ldots, p .
$$

form, in the language of [65], a contact-invariant coframe. The corresponding dual invariant differential operators $\mathcal{D}_{1}, \ldots, \mathcal{D}_{p}$ are defined by

$$
d_{H} F=\sum_{i=1}^{p}\left(\mathrm{D}_{i} F\right) d x^{i}=\sum_{i=1}^{p}\left(\mathcal{D}_{i} F\right) \omega^{i},
$$

for any differential function $F\left(x, u^{(n)}\right)$. As such, they form a system of (typically noncommuting - see (6.5) below) differential operators that map differential invariants to differential invariants.

A collection of differential invariants is called a generating set if every other differential invariant can be locally expressed as a function of them and their successive invariant derivatives. The Basis Theorem — see Theorem 7.1 below — states the existence of a finite generating set of differential invariants of any eventually freely acting Lie pseudogroup. Furthermore, the differentiated invariants $\mathcal{D}_{J} I_{\kappa}$ are not necessarily functionally independent, but may be subject to certain functional relations or differential syzygies that vanish identically:

$$
H\left(\ldots \mathcal{D}_{J} I_{\kappa} \ldots\right) \equiv 0
$$


The Syzygy Theorem 7.2 states that these all follow from a finite number of generating syzygies.

Example 5.5. Let $M=\mathbb{R}^{3}$. Consider the Lie pseudo-group $\mathcal{G}$ given by

$$
X=f(x), \quad Y=f^{\prime}(x) y+g(x), \quad U=u+\frac{f^{\prime \prime}(x) y+g^{\prime}(x)}{f^{\prime}(x)}
$$

where $f(x) \in \mathcal{D}(\mathbb{R})$ is an arbitrary local analytic diffeomorphism, while $g(x)$ is an arbitrary analytic function. We are interested in the induced action of $\mathcal{G}$ on surfaces $S \subset M$, which, for simplicity, we assume to be graphs of function $u=h(x, y)$. (The constructions can be readily adapted to parametrized surfaces.) Note that

$$
d_{H} X=f_{x} d x, \quad d_{H} Y=e_{x} d x+f_{x} d y
$$

where, for convenience, we set $e(x, y)=f^{\prime}(x) y+g(x)$, and so $e_{y}=f_{x}, f_{y}=0$. The prolonged pseudo-group transformations are found by successively applying the dual implicit differentiation operators

$$
\mathrm{D}_{X}=\frac{1}{f_{x}} \mathrm{D}_{x}-\frac{e_{x}}{f_{x}^{2}} \mathrm{D}_{y}, \quad \mathrm{D}_{Y}=\frac{1}{f_{x}} \mathrm{D}_{y}
$$

to $U=u+e_{x} / f_{x}$, whereby

$$
\begin{gathered}
U_{X}=\frac{u_{x}}{f_{x}}+\frac{e_{x x}-e_{x} u_{y}}{f_{x}^{2}}-2 \frac{f_{x x} e_{x}}{f_{x}^{3}}, \quad U_{Y}=\frac{u_{y}}{f_{x}}+\frac{f_{x x}}{f_{x}^{2}}, \\
U_{X X}=\frac{u_{x x}}{f_{x}^{2}}+\frac{e_{x x x}-e_{x x} u_{y}-2 e_{x} u_{x y}-f_{x x} u_{x}}{f_{x}^{3}}+ \\
+\frac{e_{x}^{2} u_{y y}+3 e_{x} f_{x x} u_{y}-4 e_{x x} f_{x x}-3 e_{x} f_{x x x}}{f_{x}^{4}}+8 \frac{e_{x} f_{x x}^{2}}{f_{x}^{5}}, \\
U_{X Y}=\frac{u_{x y}}{f_{x}^{2}}+\frac{f_{x x x}-f_{x x} u_{y}-e_{x} u_{y y}}{f_{x}^{3}}-2 \frac{f_{x x}^{2}}{f_{x}^{4}}, \quad U_{Y Y}=\frac{u_{y y}}{f_{x}^{2}},
\end{gathered}
$$

and so on. The prolonged pseudo-group does not act freely on $\mathrm{J}^{1}$, but is locally free and locally transitive on the open set $\left\{u_{y y} \neq 0\right\} \subset \mathrm{J}^{2}$.

To construct a moving frame, for brevity, we restrict our attention to the case $u_{y y}>0$, and use the coordinate cross-section

$$
x=y=u=u_{x}=u_{y}=0, \quad u_{x x}=u_{x y}=0, \quad u_{y y}=1, \quad u_{x^{k}}=u_{x^{k-1} y}=0, \quad k \geq 3
$$

Solving the corresponding normalization equations produces the moving frame formulae 
for the pseudo-group parameters and the corresponding phantom differential invariant:

$$
\begin{aligned}
& X=0=\iota(x), \quad f=0, \\
& Y=0=\iota(y), \quad e=0, \\
& U=0=\iota(u), \quad e_{x}=-u f_{x}, \\
& U_{Y}=0=\iota\left(u_{y}\right), \quad f_{x x}=-u_{y} f_{x}, \\
& U_{X}=0=\iota\left(u_{x}\right), \quad e_{x x}=\left(u u_{y}-u_{x}\right) f_{x}, \\
& U_{Y Y}=1=\iota\left(u_{y y}\right), \quad f_{x}=\sqrt{u_{y y}}, \\
& U_{X Y}=0=\iota\left(u_{x y}\right), \quad f_{x x x}=-\sqrt{u_{y y}}\left(u_{x y}+u u_{y y}-u_{y}^{2}\right), \\
& U_{X X}=0=\iota\left(u_{x x}\right), \quad e_{x x x}=-\sqrt{u_{y y}}\left(u_{x x}-u u_{x y}-2 u^{2} u_{y y}-2 u_{x} u_{y}+u u_{y}^{2}\right) .
\end{aligned}
$$

By this stage, we have normalized enough parameters to find the first two fundamental differential invariants of the pseudo-group, namely,

$$
J_{1}=\iota\left(u_{x y y}\right)=\frac{u_{x y y}+u u_{y y y}+2 u_{y} u_{y y}}{u_{y y}^{3 / 2}}, \quad J_{2}=\iota\left(u_{y y y}\right)=\frac{u_{y y y}}{u_{y y}^{3 / 2}},
$$

which are obtained by substituting the the moving frame normalizations (5.11) into the prolonged action formulas for $U_{X Y Y}, U_{Y Y Y}$, respectively. Further, substituting the moving frame formulae into (5.9) fixes the contact-invariant coframe

$$
\omega^{1}=\pi_{H}(\iota(d x))=\sqrt{u_{y y}} d x, \quad \omega^{2}=\pi_{H}(\iota(d y))=\sqrt{u_{y y}}(d y-u d x),
$$

and thus the dual invariant differential operators

$$
\mathcal{D}_{1}=\frac{1}{\sqrt{u_{y y}}}\left(\mathrm{D}_{x}+u \mathrm{D}_{y}\right), \quad \mathcal{D}_{2}=\frac{1}{\sqrt{u_{y y}}} \mathrm{D}_{y} .
$$

As we shall subsequently prove, all of the higher order differential invariants are obtained by successive invariant differentiation of $J_{1}, J_{2}$, which thus generate the entire algebra of differential invariants .

\section{Recurrence.}

While the invariantization respects algebraic operations, in general, the same is not true for differentiation. By a recurrence relation, we mean a formula that expresses a differentiated invariant function or form in terms of the basic differential invariants and invariant differential forms. The recurrence formulae are the most important new contribution of the equivariant moving frame method, and are the master key that unlocks the complete structure of the differential invariant algebra and, more generally, the invariant variational bicomplex for any eventually free pseudo-group action. Remarkably, the recurrence formulae can be deduced without knowledge of the explicit formulas for either the differential invariants, or the invariant differential operators, or the moving frame, or even the actual pseudo-group transformations! Indeed, they follow directly, using only linear

algebra, from the formulas for the prolonged infinitesimal generators, combined with the specification of the cross-section normalizations. 
The first step is to pull back the pseudo-group Maurer-Cartan forms to the bundle $\mathcal{H}^{(n)}$; we will continue to denote these forms by $\mu^{(n)}=\left(\ldots \mu_{B}^{a} \ldots\right)$. Let $\nu^{(n)}=$ $\left(\ldots \nu_{B}^{a} \ldots\right)$ be the one-forms on $\mathrm{J}^{n}$ obtained by pulling the latter forms back via the moving frame section $\rho: \mathrm{J}^{n} \rightarrow \mathcal{H}^{(n)}$, so $\nu_{B}^{a}=\rho^{*} \mu_{B}^{a}$. In view of Theorem 3.4, they are subject to the linear relations

$$
L^{(n)}\left(H, I, \nu^{(n)}\right)=0, \quad n \geq 0,
$$

obtained from the infinitesimal determining equations (3.15) by formally replacing the source coordinates $x^{i}, u^{\alpha}$ by the corresponding differential invariants $H^{i}=\iota\left(x^{i}\right), I^{\alpha}=$ $\iota\left(u^{\alpha}\right)$, and the vector field jet coordinates $\zeta_{B}^{a}$ by the one-forms $\nu_{B}^{a}$.

The universal recurrence formula for differential invariants and invariant differential forms of Lie pseudo-groups can now be stated; a proof can be found in [73].

Theorem 6.1. If $\Omega$ is any differential form on $\mathrm{J}^{n}$, then

$$
d \iota(\Omega)=\iota\left[d \Omega+\mathbf{v}^{(n)}(\Omega)\right]
$$

where the final term denotes the Lie derivative of $\Omega$ with respect to the prolonged infinitesimal generator $\mathbf{v}^{(n)}$, and ones uses the rule $\iota\left(\zeta_{B}^{a}\right)=\nu_{B}^{a}$ to "invariantize" the derivatives of the infinitesimal generator coefficients appearing therein.

Each phantom differential invariant is, by definition, normalized to a constant value, and hence has zero differential. As proved in [73], the corresponding phantom recurrence formulae form a system of linear algebraic equations which, provided $n \geq n_{\star}$, can be uniquely solved for the pulled-back Maurer-Cartan forms $\nu^{(n)}$. Substituting the resulting expressions into the remaining, non-phantom recurrence formulae leads to a complete system of recurrence relations. Here, we only require the horizontal components of these relations. Each horizontal pulled-back Maurer-Cartan form $\gamma_{B}^{a}=\pi_{H} \nu_{B}^{a}$ is an invariant linear combination of the contact-invariant coframe $\omega^{i}$, cf. (5.5).

In particular, choosing $\Omega=d x^{i}$ to be a basis horizontal form, the horizontal component of the resulting recurrence relation $(6.2)$ is

$$
\begin{aligned}
d_{H} \omega^{i} & \equiv d \iota\left(d x^{i}\right)=\iota\left(d\left(d x^{i}\right)+\mathbf{v}\left(d x^{i}\right)\right)=\iota\left(d \xi^{i}\right) \equiv \iota\left(\sum_{j=1}^{p} \mathrm{D}_{j} \xi^{i} d x^{j}\right) \\
& =\sum_{j=1}^{p} \iota\left(\frac{\partial \xi^{i}}{\partial x^{j}}+\sum_{\alpha=1}^{q} u_{j}^{\alpha} \frac{\partial \xi^{i}}{\partial u^{\alpha}}\right) \wedge \iota\left(d x^{j}\right) \equiv \sum_{j=1}^{p}\left(\gamma_{j}^{i}+\sum_{\alpha=1}^{q} I_{j}^{\alpha} \gamma_{\alpha}^{i}\right) \wedge \omega^{j} .
\end{aligned}
$$

Replacing the pulled back first order horizontal Maurer-Cartan forms $\gamma_{j}^{i}, \gamma_{\alpha}^{i}$, by their expressions resulting from solving the phantom recurrence formula produces the explicit recurrence formulas

$$
d_{H} \omega^{i}=-\sum_{j<k} Y_{j k}^{i} \omega^{j} \wedge \omega^{k}
$$


The commutator invariants $Y_{j k}^{i}$ appearing in (6.4) also provide the coefficients in the commutator formulae for the invariant differential operators

$$
\left[\mathcal{D}_{j}, \mathcal{D}_{k}\right]=\mathcal{D}_{j} \mathcal{D}_{k}-\mathcal{D}_{k} \mathcal{D}_{j}=\sum_{i=1}^{p} Y_{j k}^{i} \mathcal{D}_{i}
$$

Example 6.2. Return to the pseudo-group $\mathcal{G}$ treated in Example 5.5. To establish the recurrence relations for the invariantly differentiated functions and forms, we first write out its infinitesimal generator ${ }^{\dagger}$

$$
\mathbf{v}=a(x) \frac{\partial}{\partial x}+\left[y a_{x}(x)+b(x)\right] \frac{\partial}{\partial y}+\left[y a_{x x}(x)+b_{x}(x)\right] \frac{\partial}{\partial u},
$$

where $a(x), b(x)$ are arbitrary scalar functions. We prolong the vector field to the submanifold jet bundle $\mathrm{J}^{n}$ using (4.2-3); the resulting coefficients will be linear combinations of derivatives $a_{k}=\mathrm{D}_{x}^{k} a, b_{k}=\mathrm{D}_{x}^{k} b$. According to our rules, the invariantized coefficient will be the corresponding invariant linear combination of the pulled-back Maurer-Cartan forms. We use $\alpha_{k}, \beta_{k}$, to denote the horizontal constituents of the Maurer-Cartan forms corresponding to $a_{k}, b_{k}$, respectively. We do not need to compute these one-forms directly, since they will be determined from the recurrence formulae for the phantom differential invariants. Indeed, let us now write out the recurrence formulae (6.2) for the fundamental differential invariants. Here, we only display their horizontal components, using $\equiv$ to denote equality modulo contact forms. We begin with the phantom invariants

$$
\begin{aligned}
& 0=d \iota(x)=\iota(d x+a) \equiv \omega^{1}+\alpha_{0}, \\
& 0=d \iota(y)=\iota\left(d y+y a_{x}+b\right) \equiv \omega^{2}+\beta_{0} \text {, } \\
& 0=d \iota(u)=\iota(d u+\varphi) \equiv \iota\left(u_{x} d x+u_{y} d y+y a_{x x}+b_{x}\right) \equiv \beta_{1} \text {, } \\
& 0=d \iota\left(u_{x}\right)=\iota\left(d u_{x}+\varphi^{x}\right) \\
& \equiv \iota\left(u_{x x} d x+u_{x y} d y+y\left(a_{x x x}-u_{y} a_{x x}\right)+b_{x x}-u_{x} a_{x}-u_{y} b_{x}\right) \equiv \beta_{2}, \\
& 0=d \iota\left(u_{y}\right)=\iota\left(d u_{y}+\varphi^{x}\right) \equiv \iota\left(u_{x y} d x+u_{y y} d y+a_{x x}-u_{y} a_{x}\right) \equiv \omega^{2}+\alpha_{2} \text {, } \\
& 0=d \iota\left(u_{x x}\right)=\iota\left(d u_{x x}+\varphi^{x x}\right) \equiv \beta_{3} \text {, } \\
& 0=d \iota\left(u_{x y}\right)=\iota\left(d u_{x y}+\varphi^{x y}\right) \equiv J_{1} \omega^{2}-\beta_{2}+\alpha_{3} \text {, } \\
& 0=d \iota\left(u_{y y}\right)=\iota\left(d u_{y y}+\varphi^{y y}\right) \equiv J_{1} \omega^{1}+J_{2} \omega^{2}-2 \alpha_{1} \text {, } \\
& 0=d \iota\left(u_{x x x}\right)=\iota\left(d u_{x x x}+\varphi^{x x x}\right) \equiv \beta_{4} \text {, } \\
& 0=d \iota\left(u_{x x y}\right)=\iota\left(d u_{x x y}+\varphi^{x x y}\right) \equiv K_{1} \omega^{2}-2 J_{1} \beta_{1}-\beta_{2}+\alpha_{4} \text {, }
\end{aligned}
$$

and so on, where we set $K_{1}=\iota\left(u_{x x y y}\right), K_{2}=\iota\left(u_{x y y y}\right), K_{3}=\iota\left(u_{y y y y}\right)$. Solving the resulting linear system produces the formulae for the horizontal component of the pulled-

$\dagger$ Actually, to perform this computation, we only need the infinitesimal determining equations; however, employing their explicit solution, as provided in (6.6), makes the calculations more compact and easier to follow. 
back Maurer-Cartan forms:

$$
\begin{gathered}
\alpha_{0}=-\omega^{1}, \quad \alpha_{1}=\frac{1}{2} J_{1} \omega^{1}+\frac{1}{2} J_{2} \omega^{2}, \quad \alpha_{2}=-\omega^{2}, \quad \alpha_{3}=-J_{1} \omega^{2}, \\
\alpha_{4}=-K_{1} \omega^{2}, \quad \beta_{0}=-\omega^{2}, \quad \beta_{1}=\beta_{2}=\beta_{3}=\beta_{4}=0,
\end{gathered}
$$

and similarly for the higher order forms. Observe that we did not require the explicit formulas for either the moving frame map or the original Maurer-Cartan forms to deduce these expressions. Substituting these expressions into the next couple of recurrence relations

$$
\begin{aligned}
& d_{H} J_{1} \equiv d \iota\left(u_{x y y}\right)=\iota\left(d u_{x y y}+\varphi^{x y y}\right) \\
& \quad \equiv \iota\left(u_{x x y y} d x+u_{x y y y} d y-3 u_{x y y} a_{x}-u_{y y y} b_{x}-\left(2 u_{y y}+y u_{y y y}\right) a_{x x}\right) \\
& \quad=K_{1} \omega^{1}+K_{2} \omega^{2}-3 J_{1} \alpha_{1}-J_{2} \beta_{1}-2 \alpha_{2}=\left(K_{1}-\frac{3}{2} J_{1}^{2}\right) \omega^{1}+\left(K_{2}-\frac{3}{2} J_{1} J_{2}+2\right) \omega^{2}, \\
& d_{H} J_{2} \equiv d \iota\left(u_{y y y}\right)=\iota\left(d u_{y y y}+\varphi^{y y y}\right) \equiv \iota\left(u_{x y y y} d x+u_{y y y y} d y-3 u_{y y y} a_{x}\right) \\
& =K_{2} \omega^{1}+K_{3} \omega^{2}-3 J_{2} \alpha_{1}=\left(K_{2}-\frac{3}{2} J_{1} J_{2}\right) \omega^{1}+\left(K_{3}-\frac{3}{2} J_{2}^{2}\right) \omega^{2},
\end{aligned}
$$

produces the explicit recurrence formulae

$$
\begin{array}{ll}
\mathcal{D}_{1} J_{1}=K_{1}-\frac{3}{2} J_{1}^{2}, & \mathcal{D}_{2} J_{1}=K_{2}-\frac{3}{2} J_{1} J_{2}+2, \\
\mathcal{D}_{1} J_{2}=K_{2}-\frac{3}{2} J_{1} J_{2}, & \mathcal{D}_{2} J_{2}=K_{3}-\frac{3}{2} J_{2}^{2} .
\end{array}
$$

Comparing the second and third yields the fundamental differential syzygy

$$
\mathcal{D}_{1} J_{2}-\mathcal{D}_{2} J_{1}=-2
$$

among the lowest order differential invariants. Proceeding by induction, we deduce that all higher-order differential invariants are obtained by successively applying the invariant total derivative operators to the fundamental invariants $J_{1}, J_{2}$.

Similarly, we can determine the differentials of the basic invariant horizontal and contact forms. As in (6.3),

$$
\begin{aligned}
d_{H} \omega^{1} \equiv d \iota(d x)=\iota[d(d x)+\mathbf{v}(d x)] & =\iota(d a) \equiv \iota\left(a_{x} d x\right)=\alpha_{1} \wedge \omega^{1}=-\frac{1}{2} J_{2} \omega^{1} \wedge \omega^{2}, \\
d_{H} \omega^{2} \equiv d \iota(d y)=\iota[d(d y)+\mathbf{v}(d y)] & =\iota\left[d\left(y a_{x}+b\right)\right] \equiv \iota\left[\left(y a_{x x}+b_{x}\right) d x+a_{x} d y\right] \\
& =\beta_{1} \wedge \omega^{1}+\alpha_{1} \wedge \omega^{2}=\frac{1}{2} J_{1} \omega^{1} \wedge \omega^{2} .
\end{aligned}
$$

These imply the commutation formula

$$
\left[\mathcal{D}_{1}, \mathcal{D}_{2}\right]=\frac{1}{2} J_{2} \mathcal{D}_{1}-\frac{1}{2} J_{1} \mathcal{D}_{2}
$$

for the invariant differential operators. Moreover, applying (6.8) to $J_{1}$ allows us to write

$$
J_{2}=\frac{2 \mathcal{D}_{1} \mathcal{D}_{2} J_{1}-2 \mathcal{D}_{2} \mathcal{D}_{1} J_{1}+J_{1} \mathcal{D}_{2} J_{1}}{\mathcal{D}_{1} J_{1}}
$$

and hence $J_{1}$ alone suffices to generate the entire differential invariant algebra. The syzygy (6.7) then becomes an identity among the invariant derivatives of $J_{1}$, as does the modified commutation formula obtained by substituting (6.9) into (6.8). 
Example 6.3. Let us determine the algebra of differential invariants for the $\mathrm{KdV}$ symmetry group (4.10), acting on surfaces $u=h(x, y)$. The prolonged action is readily computed via implicit differentiation:

$$
\begin{gathered}
T=e^{3 \lambda_{4}}\left(t+\lambda_{1}\right), \quad X=e^{\lambda_{4}}\left(\lambda_{3} t+x+\lambda_{1} \lambda_{3}+\lambda_{2}\right), \quad U=e^{-2 \lambda_{4}}\left(u+\lambda_{3}\right), \\
U_{T}=e^{-5 \lambda_{4}}\left(u_{t}-\lambda_{3} u_{x}\right), \quad U_{X}=e^{-3 \lambda_{4}} u_{x}, \\
\left.U_{T T}=e^{-8 \lambda_{4}}\left(u_{t t}-2 \lambda_{3} u_{t x}+\lambda_{3}^{2} u_{x x}\right), \quad U_{T X}=e^{-6 \lambda_{4}}\left(u_{t x}-\lambda_{3} u_{x x}\right), \quad U_{X X}=e^{-4 \lambda_{4}} u_{x x} .10\right)
\end{gathered}
$$

Let us work with the coordinate cross-section

$$
t=x=u=0, \quad u_{t}=1, \quad u_{t}+u u_{x}>0 .
$$

Solving the corresponding normalization equations $T=0, \quad X=0, \quad U=0, \quad U_{T}=1$, produces the moving frame

$$
\lambda_{1}=-t, \quad \lambda_{2}=-x, \quad \lambda_{3}=-u, \quad \lambda_{4}=\frac{1}{5} \log \left(u_{t}+u u_{x}\right) .
$$

The differential invariants are obtained by substituting (6.11) into (6.10):

$$
\begin{aligned}
& H^{1}=\iota(t)=0, \quad H^{2}=\iota(x)=0, \quad I_{0}=\iota(u)=0, \quad I_{10}=\iota\left(u_{t}\right)=1, \\
& I_{01}=\iota\left(u_{x}\right)=\frac{u_{x}}{\left(u_{t}+u u_{x}\right)^{3 / 5}}, \quad I_{20}=\iota\left(u_{t t}\right)=\frac{u_{t t}+2 u u_{t x}+u^{2} u_{x x}}{\left(u_{t}+u u_{x}\right)^{8 / 5}}, \\
& I_{11}=\iota\left(u_{t x}\right)=\frac{u_{t x}+u u_{x x}}{\left(u_{t}+u u_{x}\right)^{6 / 5}}, \quad I_{02}=\iota\left(u_{x x}\right)=\frac{u_{x x}}{\left(u_{t}+u u_{x}\right)^{4 / 5}}, \\
& I_{30}=\iota\left(u_{t t t}\right)=\frac{u_{t t t}+3 u u_{t t x}+3 u^{2} u_{t x x}+u^{3} u_{x x x}}{\left(u_{t}+u u_{x}\right)^{11 / 5}}, \\
& I_{21}=\iota\left(u_{t t x}\right)=\frac{u_{t t x}+2 u u_{t x x}+u^{2} u_{x x x}}{\left(u_{t}+u u_{x}\right)^{9 / 5}}, \\
& I_{12}=\iota\left(u_{t x x}\right)=\frac{u_{t x x}+u u_{x x x}}{\left(u_{t}+u u_{x}\right)^{7 / 5}}, \quad I_{03}=\iota\left(u_{x x x}\right)=\frac{u_{x x x}}{u_{t}+u u_{x}},
\end{aligned}
$$

The Replacement Rule (5.3) allows us to immediately rewrite the KdV equation in terms of the differential invariants by applying the invariantization process

$$
0=\iota\left(u_{t}+u u_{x}+u_{x x x}\right)=1+I_{03}=\frac{u_{t}+u u_{x}+u_{x x x}}{u_{t}+u u_{x}} .
$$

The invariantized horizontal coframe

$$
\begin{aligned}
& \omega^{1}=\pi_{H} \iota(d t)=\left(u_{t}+u u_{x}\right)^{3 / 5} d t, \\
& \omega^{2}=\pi_{H} \iota(d x)=-u\left(u_{t}+u u_{x}\right)^{1 / 5} d t+\left(u_{t}+u u_{x}\right)^{1 / 5} d x,
\end{aligned}
$$

produces the invariant differential operators

$$
\mathcal{D}_{1}=\left(u_{t}+u u_{x}\right)^{-3 / 5} \mathrm{D}_{t}+u\left(u_{t}+u u_{x}\right)^{-3 / 5} \mathrm{D}_{x}, \quad \mathcal{D}_{2}=\left(u_{t}+u u_{x}\right)^{-1 / 5} \mathrm{D}_{x} .
$$

The commutation formula

$$
\left[\mathcal{D}_{1}, \mathcal{D}_{2}\right]=\frac{3}{5}\left(I_{11}+I_{01}^{2}\right) \mathcal{D}_{1}-\frac{1}{5}\left(I_{20}+6 I_{01}\right) \mathcal{D}_{2}
$$


can be found either directly or through recurrence as in (6.4). The recurrence relations

$$
\begin{array}{ll}
\mathcal{D}_{1} I_{01}=I_{11}-\frac{3}{5} I_{01}^{2}-\frac{3}{5} I_{01} I_{20}, & \mathcal{D}_{2} I_{01}=I_{02}-\frac{3}{5} I_{01}^{3}-\frac{3}{5} I_{01} I_{11}, \\
\mathcal{D}_{1} I_{20}=I_{30}+2 I_{11}-\frac{8}{5} I_{01} I_{20}-\frac{8}{5} I_{20}^{2}, & \mathcal{D}_{2} I_{20}=I_{21}+2 I_{01} I_{11}-\frac{8}{5} I_{01}^{2} I_{20}-\frac{8}{5} I_{11} I_{20}, \\
\mathcal{D}_{1} I_{11}=I_{21}+I_{02}-\frac{6}{5} I_{01} I_{11}-\frac{6}{5} I_{11} I_{20}, & \mathcal{D}_{2} I_{11}=I_{12}+I_{01} I_{02}-\frac{6}{5} I_{01}^{2} I_{11}-\frac{6}{5} I_{11}^{2}, \\
\mathcal{D}_{1} I_{02}=I_{12}-\frac{4}{5} I_{01} I_{02}-\frac{4}{5} I_{02} I_{20}, & \mathcal{D}_{2} I_{02}=I_{03}-\frac{4}{5} I_{01}^{2} I_{02}-\frac{4}{5} I_{02} I_{11},
\end{array}
$$

and so on, can be derived using (6.2) as in the previous example. They imply that all higher order differential invariants can be constructed by repeatedly applying the invariant differential operators to the generating invariants $I_{01}, I_{20}$. Moreover, applying the commutation identity (6.14) to $I_{01}$ and one of its derivatives, e.g., $\mathcal{D}_{1} I_{01}$, produces

$$
\begin{aligned}
\mathcal{D}_{1} \mathcal{D}_{2} I_{01}-\mathcal{D}_{2} \mathcal{D}_{1} I_{01} & =\frac{3}{5}\left(I_{11}+I_{01}^{2}\right) \mathcal{D}_{1} I_{01}-\frac{1}{5}\left(I_{20}+6 I_{01}\right) \mathcal{D}_{2} I_{01}, \\
\mathcal{D}_{1} \mathcal{D}_{2} \mathcal{D}_{1} I_{01}-\mathcal{D}_{2} \mathcal{D}_{1}^{2} I_{01} & =\frac{3}{5}\left(I_{11}+I_{01}^{2}\right) \mathcal{D}_{1}^{2} I_{01}-\frac{1}{5}\left(I_{20}+6 I_{01}\right) \mathcal{D}_{2} \mathcal{D}_{1} I_{01}
\end{aligned}
$$

We regard this as a pair of linear algebraic equations, which can be solved for $I_{20}$ and $I_{11}$ as rational combinations of derivatives of $I_{01}$. We conclude that the single differential invariant $I_{01}$ generates the entire algebra of $\mathrm{KdV}$ differential invariants.

\section{The Algebra of Differential Invariants.}

The algebra of differential invariants of an eventually free Lie pseudo-group is a noncommutative differential algebra whose entire structure follows from the recurrence formulae for the differentiated invariants. To establish constructive versions of the fundamental Basis and Syzygy Theorems, we appeal to techniques from computational algebra, specifically Gröbner bases, [22], which rely on imposing term orderings on the relevant polynomial modules. An issue worth investigation is the intelligent selection of term orderings for practical computations.

There are two important commutative algebraic modules associated with a prolonged pseudo-group action on submanifold jets. At each $z \in M$, let $\left.\mathcal{I}\right|_{z}$ denote the symbol module, $[\mathbf{1 1}, \mathbf{8 3}]$, of the linearized determining system (3.15). As a consequence of involutivity, at each $z \in M$, the symbols span a submodule $\left.\mathcal{I}\right|_{z} \subset \mathcal{T}$ of the $\mathbb{R}[t]$ module $\mathcal{T} \simeq \mathbb{R}[t] \otimes \mathbb{R}^{m}$ consisting of algebraic polynomials $\eta(t, T)=\sum \eta_{a}(t) T^{a}$ depending on $t=\left(t_{1}, \ldots, t_{m}\right)$ and linearly on $T=\left(T^{1}, \ldots, T^{m}\right)$. Explicitly, the symbol maps the vector field jet coordinate $\zeta_{B}^{a}$ for $B=\left(b_{1}, \ldots, b_{k}\right)$ to the monomial $t_{B} T^{a}=t_{b_{1}} \cdots t_{b_{k}} T^{a}$. The symbol of a linear function of the vector field jets is the polynomial constructed by applying the linear symbol map to its highest order terms.

Analogously, let $\mathcal{S} \simeq \mathbb{R}[s] \otimes \mathbb{R}^{q}$ denote the $\mathbb{R}[s]$ module consisting of polynomials $\sigma(s, S)=\sum_{\alpha=1}^{q} \sigma_{\alpha}(s) S^{\alpha}$ depending on $s=\left(s_{1}, \ldots, s_{p}\right)$ and linearly on $S=\left(S^{1}, \ldots, S^{q}\right)$. At each submanifold 1 -jet $z^{(1)}=\left(x, u^{(1)}\right)=\left(\ldots x^{i} \ldots u^{\alpha} \ldots u_{i}^{\alpha} \ldots\right) \in \mathrm{J}^{1}$, we define a linear $\operatorname{map} \boldsymbol{\beta}: \mathbb{R}^{m} \times \mathbb{R}^{m} \rightarrow \mathbb{R}^{m}$ by $(s, S)=\boldsymbol{\beta}\left(z^{(1)} ; t, T\right)$, with components

$$
s_{i}=t_{i}+\sum_{\alpha=1}^{q} u_{i}^{\alpha} t_{p+\alpha}, \quad S^{\alpha}=T^{p+\alpha}-\sum_{i=1}^{p} u_{i}^{\alpha} T^{i} .
$$


The linear map $\boldsymbol{\beta}$ acts on symbol polynomials via pull-back:

$$
\boldsymbol{\beta}^{*}[\sigma(s, S)]=\sigma\left(\boldsymbol{\beta}\left(z^{(1)} ; t, T\right)\right) .
$$

The prolonged symbol module $\left.\mathcal{J}\right|_{z^{(1)}} \subset \mathcal{S}$ at $z^{(1)} \in \mathrm{J}^{1}$ is defined as the inverse image of the symbol module $\left.\mathcal{I}\right|_{z} \subset \mathcal{T}$ at $z=\pi_{0}^{1}\left(z^{(1)}\right)$ under the pull-back map:

$$
\left.\mathcal{J}\right|_{z^{(1)}}=\left(\boldsymbol{\beta}^{*}\right)^{-1}\left(\left.\mathcal{I}\right|_{z}\right)=\left\{\sigma\left|\boldsymbol{\beta}^{*}(\sigma) \in \mathcal{I}\right|_{z}\right\} .
$$

The invariantization process associated with a moving frame acts coefficient-wise on prolonged symbol polynomials, taking

$$
\sigma\left(x, u^{(1)} ; s, S\right)=\sum_{\alpha=1}^{q} \sum_{\# J \geq 0} h_{\alpha}^{J}\left(x, u^{(1)}\right) s_{J} S^{\alpha},
$$

say, to

$$
\widetilde{\sigma}\left(H, I^{(1)} ; s, S\right)=\iota\left[\sigma\left(x, u^{(1)} ; s, S\right)\right]=\sum_{\alpha=1}^{q} \sum_{\# J \geq 0} h_{\alpha}^{J}\left(H, I^{(1)}\right) s_{J} S^{\alpha} .
$$

We let $\left.\widetilde{\mathcal{J}}\right|_{\left(H, I^{(1)}\right)}=\iota\left(\left.\mathcal{J}\right|_{\left(x, u^{(1)}\right)}\right)$ denote the invariantized prolonged symbol module, and $\left.\widetilde{\mathcal{J}}^{>n_{\star}}\right|_{\left(H, I^{(1)}\right)}$ the submodule containing those polynomials that have degree $>n_{\star}$, the order of freeness, in the $s$ 's. In particular, if $\mathcal{G}$ acts transitively on $J^{1}$ and we use a minimal order moving frame, then the fundamental differential invariants $\left(H, I^{(1)}\right)$ are all constant, and so the invariantized prolonged symbol module is independent of the jet.

We identify the invariantized polynomial (7.3) with the differential invariant

$$
I_{\tilde{\sigma}}=\sum_{\alpha=1}^{q} \sum_{\# J \geq 0} h_{\alpha}^{J}\left(H, I^{(1)}\right) I_{J}^{\alpha} .
$$

This collection of differential invariants turns out to be much better adapted to the structure of the differential invariant algebra, since their recurrence formulae (6.2) can be shown to take the form

$$
\mathcal{D}_{i} I_{\tilde{\sigma}}=I_{s_{i} \tilde{\sigma}}+M_{\tilde{\sigma}, i},
$$

in which, when $\operatorname{deg} \widetilde{\sigma}>n_{\star}$, the leading term $I_{s_{i} \tilde{\sigma}}$ is strictly of higher order than the correction term $M_{\tilde{\sigma}, i}$. Iteration of (7.5) underlies the proof of a Constructive Basis Theorem for the differential invariant algebra, [74].

Theorem 7.1. Let $\mathcal{G}$ be a Lie pseudo-group that acts freely an open subset of the submanifold jet bundle $\mathrm{J}^{n_{\star}}$ for some $n_{\star}>0$. Then a finite generating system for its differential invariant algebra consists of:

- the differential invariants $I_{1}=I_{\sigma_{1}}, \ldots, I_{l}=I_{\sigma_{l}}$, where $\sigma_{1}, \ldots, \sigma_{l}$ form a Gröbner basis for the invariantized prolonged symbol submodule $\widetilde{\mathcal{J}}^{>n_{\star}}$, and, possibly,

- a finite number of additional differential invariants of order $\leq n_{\star}$. 
We are also able to exhibit a finite generating system of differential syzygies. First, the commutator formulae (6.5) for the invariant differential operators imply the commutator syzygies

$$
\mathcal{D}_{J} I_{\tilde{\sigma}}-\mathcal{D}_{\tilde{J}} I_{\tilde{\sigma}}=M_{\tilde{\sigma}, J}-M_{\tilde{\sigma}, \tilde{J}} \equiv N_{J, \tilde{J}, \tilde{\sigma}}, \quad \text { whenever } \quad \widetilde{J}=\pi(J)
$$

for some permutation $\pi$. Provided $\operatorname{deg} \widetilde{\sigma}>n_{\star}$, the right hand side $N_{J, \tilde{J}, \tilde{\sigma}}$ has strictly lower order than the terms on the left hand side. Technically, there are an infinite number of algebraically independent commutator syzygies, although they are all consequences of the two-sided ideal of invariant differential operators that is finitely generated by the original commutator identities (6.5).

In addition, any algebraic syzygy that is satisfied by the Gröbner basis polynomials of the prolonged symbol module provides an additional differential syzygy amongst the generating invariants. In detail, to each invariantly parametrized polynomial

$$
q\left(H, I^{(1)} ; s\right)=\sum_{J} q_{J}\left(H, I^{(1)}\right) s_{J} \in \mathbb{R}[s]
$$

we associate an invariant differential operator

$$
q\left(H, I^{(1)} ; \mathcal{D}\right)=\sum_{J} q_{J}\left(H, I^{(1)}\right) \mathcal{D}_{J},
$$

where we adopt the normal ordering convention that the latter sum ranges over nondecreasing multi-indices $j_{1} \leq j_{2} \leq \cdots \leq j_{k}$. In view of $(7.5)$, whenever $\widetilde{\sigma}\left(H, I^{(1)} ; s, S\right) \in$ $\left.\widetilde{\mathcal{J}}\right|_{\left(H, I^{(1)}\right)}$, we can write

$$
q\left(H, I^{(1)} ; \mathcal{D}\right) I_{\tilde{\sigma}\left(H, I^{(1)} ; s, S\right)}=I_{q\left(H, I^{(1)} ; s\right) \tilde{\sigma}\left(H, I^{(1)} ; s, S\right)}+R_{q, \tilde{\sigma}},
$$

where $R_{q, \tilde{\sigma}}$ has strictly lower order. Thus, any algebraic syzygy

$$
\sum_{\nu=1}^{l} q_{\nu}\left(H, I^{(1)}, s\right) \sigma_{\nu}\left(H, I^{(1)} ; s, S\right)=0
$$

among the Gröbner basis polynomials of the invariantized prolonged symbol module induces a syzygy among the generating differential invariants,

$$
\sum_{\nu=1}^{l} q_{\nu}\left(H, I^{(1)}, \mathcal{D}\right) I_{\tilde{\sigma}_{\nu}}=\widehat{R}_{q, \tilde{\sigma}}
$$

whose right hand side is of strictly lower order. By combining these constituents, we deduce a general, constructive Syzygy Theorem for differential invariant algebras of eventually free Lie pseudo-groups, [74].

Theorem 7.2. Every differential syzygy among the generating differential invariants is a combination of the following:

- the syzygies among the differential invariants of order $\leq n_{\star}$,

- the commutator syzygies,

- syzygies arising from an algebraic syzygy among the Gröbner basis polynomials. 
Example 7.3. For the pseudo-group treated in Example 5.5, recall that the order of freeness is $n_{\star}=2$. Since $\mathcal{G}$ acts transitively on an open subset of $\mathrm{J}^{1}$, we can ignore the dependence of the invariantized symbol polynomials, etc., on $\left(H, I^{(1)}\right)=$ const. In view of the cross-section equations (5.10), the prolonged symbol submodule $\mathcal{J}^{>2}$ is spanned by the monomials $s_{1}^{i} s_{2}^{j} S$ for $i+j \geq 3, j \geq 2$. Thus, the Gröbner basis consists of the monomials

$$
\tilde{\sigma}_{1}(s, S)=s_{1} s_{2}^{2} S, \quad \widetilde{\sigma}_{2}(s, S)=s_{2}^{3} S,
$$

whose corresponding differential invariants $J_{1}=I_{1,2}, J_{2}=I_{0,3}$, appear in (5.12). Since there are no low order differential invariants, Theorem 7.1 immediately implies that $J_{1}, J_{2}$ generate the differential invariant algebra. (However, as we learned, they do not form a minimal generating set.) Furthermore, there is a single generating syzygy among the Gröbner basis polynomials, namely,

$$
s_{2} \widetilde{\sigma}_{1}-s_{1} \widetilde{\sigma}_{2}=0
$$

which corresponds to the basic differential syzygy (6.7). Theorem 7.2 implies that the syzygies among the differentiated invariants are all differential consequences of it and the commutation relation $(6.8)$.

Acknowledgments: Thanks to Francis Valiquette for corrections and comments. 


\section{References}

[1] Anderson, I.M., The Variational Bicomplex, Utah State Technical Report, 1989, http://math.usu.edu/ f fg_mp.

[2] Anderson, I.M., Introduction to the variational bicomplex, Contemp. Math. 132 (1992), 51-73.

[3] Arnol'd, V.I., Sur la géométrie différentielle des groupes de Lie de dimension infinie et ses applications à l'hydrodynamique des fluides parfaits, Ann. Inst. Fourier Grenoble 16 (1966), 319-361.

[4] Bazin, P.-L., and Boutin, M., Structure from motion: theoretical foundations of a novel approach using custom built invariants, SIAM J. Appl. Math. 64 (2004), $1156-1174$.

[5] Berchenko, I.A., and Olver, P.J., Symmetries of polynomials, J. Symb. Comp. 29 (2000), 485-514.

[6] Bílă, N., Mansfield, E.L., and Clarkson, P.A., Symmetry group analysis of the shallow water and semi-geostrophic equations, Quart. J. Mech. Appl. Math. 59 (2006), 95-123.

[7] Bleecker, D., Gauge Theory and Variational Principles, Addison-Wesley Publ. Co., Reading, Mass., 1981.

[8] Boutin, M., Numerically invariant signature curves, Int. J. Computer Vision 40 (2000), 235-248.

[9] Boutin, M., On orbit dimensions under a simultaneous Lie group action on $n$ copies of a manifold, J. Lie Theory 12 (2002), 191-203.

[10] Boyko, V., Patera, J., and Popovych, R., Computation of invariants of Lie algebras by means of moving frames, J. Phys. A 39 (2006), 5749-5762.

[11] Bryant, R.L., Chern, S.-S., Gardner, R.B., Goldschmidt, H.L., and Griffiths, P.A., Exterior Differential Systems, Math. Sci. Res. Inst. Publ., vol. 18, Springer-Verlag, New York, 1991.

[12] Calabi, E., Olver, P.J., Shakiban, C., Tannenbaum, A., and Haker, S., Differential and numerically invariant signature curves applied to object recognition, Int. J. Computer Vision 26 (1998), 107-135.

[13] Cantwell, B.J., Introduction to Symmetry Analysis, Cambridge University Press, Cambridge, 2003.

[14] Cartan, É., La Méthode du Repère Mobile, la Théorie des Groupes Continus, et les Espaces Généralisés, Exposés de Géométrie, no. 5, Hermann, Paris, 1935.

[15] Cartan, É., Les Systèmes Différentiels Extérieurs et leurs Applications Géométriques, Exposés de Géométrie, no. 14, Hermann, Paris, 1945.

[16] Cartan, É., Les sous-groupes des groupes continus de transformations, in: Oeuvres Complètes, part. II, vol. 2, Gauthier-Villars, Paris, 1953, pp. 719-856.

[17] Cartan, É., Sur la structure des groupes infinis de transformations, in: Oeuvres Complètes, Part. II, Vol. 2, Gauthier-Villars, Paris, 1953, pp. 571-714.

[18] Cartan, É., La structure des groupes infinis, in: Oeuvres Complètes, part. II, vol. 2, Gauthier-Villars, Paris, 1953, pp. 1335-1384. 
[19] Cheh, J., Olver, P.J., and Pohjanpelto, J., Maurer-Cartan equations for Lie symmetry pseudo-groups of differential equations, J. Math. Phys. 46 (2005), 023504 .

[20] Cheh, J., Olver, P.J., and Pohjanpelto, J., Algorithms for differential invariants of symmetry groups of differential equations, Found. Comput. Math. 8 (2008), 501-532.

[21] Chern, S.S., and Moser, J.K., Real hypersurfaces in complex manifolds, Acta Math. 133 (1974), 219-271; also Selected Papers, vol. 3, Springer-Verlag, New York, 1989, pp. 209-262.

[22] Cox, D., Little, J., and O'Shea, D., Ideals, Varieties, and Algorithms, 2nd ed., Springer-Verlag, New York, 1996.

[23] David, D., Kamran, N., Levi, D., and Winternitz, P., Subalgebras of loop algebras and symmetries of the Kadomtsev-Petviashivili equation, Phys. Rev. Lett. 55 (1985), 2111-2113.

[24] Di Francesco, P., Mathieu, P., and Sénéchal, D., Conformal Field Theory, Springer-Verlag, New York, 1997.

[25] Ehresmann, C., Introduction à la théorie des structures infinitésimales et des pseudo-groupes de Lie, in: Géometrie Différentielle, Colloq. Inter. du Centre Nat. de la Rech. Sci., Strasbourg, 1953, pp. 97-110.

[26] Fefferman, C., and Graham, C.R., Conformal invariants, in: Élie Cartan et les Mathématiques d'aujourd'hui, Astérisque, hors série, Soc. Math. France, Paris, 1985, pp. 95-116.

[27] Fels, M., and Olver, P.J., Moving coframes. I. A practical algorithm, Acta Appl. Math. 51 (1998), 161-213.

[28] Fels, M., and Olver, P.J., Moving coframes. II. Regularization and theoretical foundations, Acta Appl. Math. 55 (1999), 127-208.

[29] Fuchs, D.B., Gabrielov, A.M., and Gel'fand, I.M., The Gauss-Bonnet theorem and Atiyah-Patodi-Singer functionals for the characteristic classes of foliations, Topology 15 (1976), 165-188.

[30] Guggenheimer, H.W., Differential Geometry, McGraw-Hill, New York, 1963.

[31] Hubert, E., Differential invariants of a Lie group action: syzygies on a generating set, J. Symb. Comp. 44 (2009), 382-416.

[32] Hubert, E., and Kogan, I.A., Rational invariants of a group action. Construction and rewriting, J. Symb. Comp. 42 (2007), 203-217.

[33] Hubert, E., and Kogan, I.A., Smooth and algebraic invariants of a group action. Local and global constructions, Found. Comput. Math. 7 (2007), 455-493.

[34] Itskov, V., Orbit Reduction of Exterior Differential Systems, Ph.D. Thesis, University of Minnesota, 2002.

[35] Itskov, V., Olver, P.J., and Valiquette, F., Lie completion of pseudo-groups, preprint, University of Minnesota, 2009.

[36] Kamran, N., Contributions to the study of the equivalence problem of Elie Cartan and its applications to partial and ordinary differential equations, Mém. Cl. Sci. Acad. Roy. Belg. 45 (1989), Fac. 7. 
[37] Kamran, N., and Robart, T., A manifold structure for analytic isotropy Lie pseudogroups of infinite type, J. Lie Theory 11 (2001), 57-80.

[38] Kim, P., Invariantization of Numerical Schemes for Differential Equations Using Moving Frames, Ph.D. Thesis, University of Minnesota, Minneapolis, 2006.

[39] Kogan, I.A., and Olver, P.J., Invariant Euler-Lagrange equations and the invariant variational bicomplex, Acta Appl. Math. 76 (2003), 137-193.

[40] Kruglikov, B., and Lychagin, V., Invariants of pseudogroup actions: homological methods and finiteness theorem, Int. J. Geom. Methods Mod. Phys. 3 (2006), 1131-1165.

[41] Kumpera, A., Invariants différentiels d'un pseudogroupe de Lie, J. Diff. Geom. 10 (1975), 289-416.

[42] Kuranishi, M., On E. Cartan's prolongation theorem of exterior differential systems, Amer. J. Math. 79 (1957), 1-47.

[43] Kuranishi, M., On the local theory of continuous infinite pseudo groups I, Nagoya Math. J. 15 (1959), 225-260.

[44] Kuranishi, M., On the local theory of continuous infinite pseudo groups II, Nagoya Math. J. 19 (1961), 55-91.

[45] Lie, S., Über unendlichen kontinuierliche Gruppen, Christ. Forh. Aar. 8 (1883), 1-47; also Gesammelte Abhandlungen, Vol. 5, B.G. Teubner, Leipzig, 1924, pp. $314-360$.

[46] Lie, S., Die Grundlagen für die Theorie der unendlichen kontinuierlichen Transformationsgruppen, Leipzig. Ber. 43 (1891), 316-393; also Gesammelte Abhandlungen, Vol. 6, B.G. Teubner, Leipzig, 1927, pp. 300-364.

[47] Lisle, I.G., and Reid, G.J., Cartan structure of infinite Lie pseudogroups, in: Geometric Approaches to Differential Equations, P.J. Vassiliou and I.G. Lisle, eds., Austral. Math. Soc. Lect. Ser., 15, Cambridge University Press, Cambridge, 2000, pp. 116-145.

[48] Mackenzie, K., General Theory of Lie Groupoids and Lie Algebroids, London Math. Soc. Lecture Notes, vol. 213, Cambridge University Press, Cambridge, 2005.

[49] Mansfield, E.L., Algorithms for symmetric differential systems, Found. Comput. Math. 1 (2001), 335-383.

[50] Marí Beffa, G., Relative and absolute differential invariants for conformal curves, $J$. Lie Theory 13 (2003), 213-245.

[51] Marí Beffa, G., Poisson geometry of differential invariants of curves in some nonsemisimple homogeneous spaces, Proc. Amer. Math. Soc. 134 (2006), 779-791.

[52] Marí-Beffa, G., and Olver, P.J., Differential invariants for parametrized projective surfaces, Commun. Anal. Geom. 7 (1999), 807-839.

[53] Martina, L., Sheftel, M.B., and Winternitz, P., Group foliation and non-invariant solutions of the heavenly equation, J. Phys. A 34 (2001), 9243-9263.

[54] McDuff, D., and Salamon, D., Introduction to Symplectic Topology, Oxford University Press, Oxford, 1995. 
[55] McLachlan, R.I., and Quispel, G.R.W., What kinds of dynamics are there? Lie pseudogroups, dynamical systems and geometric integration, Nonlinearity 14 (2001), 1689-1705.

[56] McLenaghan, R.G., and Smirnov, R.G., Hamilton-Jacobi theory via Cartan Geometry, World Scientific, Singapore, to appear.

[57] McLenaghan, R.G., Smirnov, R.G., and The, D., An extension of the classical theory of algebraic invariants to pseudo-Riemannian geometry and Hamiltonian mechanics, J. Math. Phys. 45 (2004), 1079-1120.

[58] Medolaghi, P., Classificazione delle equazioni alle derivate parziali del secondo ordine, che ammettono un gruppo infinito di trasformazioni puntuali, Ann. Mat. Pura Appl. 1 (3) (1898), 229-263.

[59] Morozov, O., Moving coframes and symmetries of differential equations, J. Phys. A 35 (2002), 2965-2977.

[60] Morozov, O.I., Structure of symmetry groups via Cartan's method: survey of four approaches, SIGMA: Symmetry Integrability Geom. Methods Appl. 1 (2005), paper 006.

[61] Muñoz, J., Muriel, F.J., and Rodríguez, J., On the finiteness of differential invariants, J. Math. Anal. Appl. 284 (2003), 266-282.

[62] Nutku, Y., and Sheftel, M.B., Differential invariants and group foliation for the complex Monge-Ampère equation, J. Phys. A 34 (2001), 137-156.

[63] Olver, P.J., Symmetry groups and group invariant solutions of partial differential equations, J. Diff. Geom. 14 (1979), 497-542.

[64] Olver, P.J., Applications of Lie Groups to Differential Equations, Second Edition, Graduate Texts in Mathematics, vol. 107, Springer-Verlag, New York, 1993.

[65] Olver, P.J., Equivalence, Invariants, and Symmetry, Cambridge University Press, Cambridge, 1995.

[66] Olver, P.J., Classical Invariant Theory, London Math. Soc. Student Texts, vol. 44, Cambridge University Press, Cambridge, 1999.

[67] Olver, P.J., Joint invariant signatures, Found. Comput. Math. 1 (2001), 3-67.

[68] Olver, P.J., Geometric foundations of numerical algorithms and symmetry, Appl. Alg. Engin. Commun. Comput. 11 (2001), 417-436.

[69] Olver, P.J., Generating differential invariants, J. Math. Anal. Appl. 333 (2007), 450-471.

[70] Olver, P.J., Invariant submanifold flows, J. Phys. A 41 (2008), 344017.

[71] Olver, P.J., Differential invariants of surfaces, Diff. Geom. Appl. 27 (2009), 230-239.

[72] Olver, P.J., and Pohjanpelto, J., Maurer-Cartan forms and the structure of Lie pseudo-groups, Selecta Math. 11 (2005), 99-126.

[73] Olver, P.J., and Pohjanpelto, J., Moving frames for Lie pseudo-groups, Canadian J. Math. 60 (2008), 1336-1386.

[74] Olver, P.J., and Pohjanpelto, J., Differential invariant algebras of Lie pseudo-groups, Adv. Math. 222 (2009), 1746-1792.

[75] Olver, P.J., and Pohjanpelto, J., Persistence of freeness for pseudo-group actions, preprint, University of Minnesota, 2009. 
[76] Olver, P.J., Pohjanpelto, J., and Valiquette, F., On the structure of Lie pseudo-groups, SIGMA 5 (2009), 077.

[77] Ovsiannikov, L.V., Group Analysis of Differential Equations, Academic Press, New York, 1982.

[78] Pommaret, J.F., Systems of Partial Differential Equations and Lie Pseudogroups, Gordon and Breach, New York, 1978.

[79] Reinhart, B.L., Differential Geometry of Foliations: the Fundamental Integrability Problem, Springer-Verlag, New York, 1983.

[80] Riordan, J., An Introduction to Combinatorial Analysis, Princeton University Press, Princeton, N.J., 1980.

[81] Robart, T., and Kamran, N., Sur la théorie locale des pseudogroupes de transformations continus infinis I, Math. Ann. 308 (1997), 593-613.

[82] Salmon, R., Lectures on Geophysical Fluid Dynamics, Oxford University Press, Oxford, 1998.

[83] Seiler, W.M., Involution: The Formal Theory of Differential Equations and its Applications in Computer Algebra, Algorithms and Computation in Mathematics, vol. 24, Springer-Verlag, New York, 2010.

[84] Serra, J., Image Analysis and Mathematical Morphology, Academic Press, London 1982.

[85] Shemyakova, E., and Mansfield, E.L., Moving frames for Laplace invariants, in: Proceedings ISSAC2008, D. Jeffrey, ed., ACM, New York, 2008, pp. 295-302.

[86] Singer, I.M., and Sternberg, S., The infinite groups of Lie and Cartan. Part I (the transitive groups), J. Analyse Math. 15 (1965), 1-114.

[87] Spencer, D.C., Deformations of structures on manifolds defined by transitive pseudo-groups I, II, Ann. Math. 76 (1962), 306-445.

[88] Stormark, O., Lie's Structural Approach to PDE Systems, Cambridge University Press, Cambridge, 2000.

[89] Tresse, A., Sur les invariants différentiels des groupes continus de transformations, Acta Math. 18 (1894), 1-88.

[90] Tsujishita, T., On variational bicomplexes associated to differential equations, Osaka J. Math. 19 (1982), 311-363.

[91] Valiquette, F., Applications of Moving Frames to Lie pseudo-groups, Ph.D. Thesis, University of Minnesota, 2009.

[92] Valiquette, F., Structure equations of Lie pseudo-groups, J. Lie Theory 18 (2008), 869-895.

[93] Vessiot, E., Sur l'intégration des systèmes différentiels qui admettent des groupes continues de transformations, Acta. Math. 28 (1904), 307-349.

[94] Weinstein, A., Groupoids: unifying internal and external symmetry. A tour through some examples, Notices Amer. Math. Soc. 43 (1996), 744-752.

[95] Welk, M., Kim, P., and Olver, P.J., Numerical invariantization for morphological PDE schemes, in: Scale Space and Variational Methods in Computer Vision, F. Sgallari, A. Murli and N. Paragios, eds., Lecture Notes in Computer Science, vol. 4485, Springer-Verlag, New York, 2007, pp. 508-519. 